\title{
Spontaneous Neuronal Oscillations in the Human Insula are Hierarchically Organized Traveling Waves
}

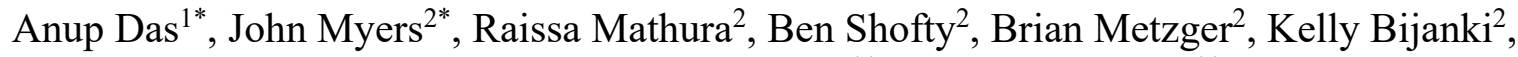 \\ Chengyuan $\mathrm{Wu}^{3}$, Joshua Jacobs ${ }^{1 * *}$, Sameer A. Sheth ${ }^{2 * *}$ \\ ${ }^{1}$ Department of Biomedical Engineering, Columbia University, New York, NY 10027 \\ ${ }^{2}$ Department of Neurosurgery, Baylor College of Medicine, Houston, TX 77030 \\ ${ }^{3}$ Department of Neurosurgery, Thomas Jefferson University, Philadelphia, PA 19107 \\ *These authors contributed equally to this work \\ **Joint senior authors
}

Correspondence:

Anup Das (ad3772@,columbia.edu), Joshua Jacobs (joshua.jacobs@columbia.edu), Department of Biomedical Engineering, Columbia University,

New York, NY 10027

John Myers (John.Myers@bcm.edu), Sameer A. Sheth (Sameer.Sheth@,bcm.edu), Department of Neurosurgery, Baylor College of Medicine, Houston, TX 77030 


\begin{abstract}
The insula plays a fundamental role in a wide range of adaptive human behaviors, but its electrophysiological dynamics are poorly understood. Here we used human intracranial electroencephalographic recordings to investigate the electrophysiological properties and hierarchical organization of spontaneous neuronal oscillations within the insula. We analyzed the neuronal oscillations of the insula directly and found that rhythms in the theta and beta frequency oscillations are widespread and spontaneously present. These oscillations are largely organized along the anterior-posterior axis of the insula. Both the left and right insula showed anterior-toposterior decreasing gradients for the power of oscillations in the beta frequency band. The left insula also showed a posterior-to-anterior decreasing frequency gradient and an anterior-toposterior decreasing power gradient in the theta frequency band. In addition to measuring the power of these oscillations, we also examined the phase of these signals across simultaneous recording channels and found that the insula oscillations in the theta and beta bands are traveling waves. The strength of the traveling waves in each frequency was positively correlated with the amplitude of each oscillation. However, the theta and beta traveling waves were uncoupled to each other in terms of phase and amplitude, which suggested that insula traveling waves in the theta and beta bands operate independently. Our findings provide new insights into the spatiotemporal dynamics and hierarchical organization of neuronal oscillations within the insula, which, given its rich connectivity with widespread cortical regions, indicates that oscillations and traveling waves have an important role in intra- and inter-insula communication.
\end{abstract}




\section{Introduction}

The insula plays a critical role in cognitive control (Vinod Menon \& Uddin, 2010), awareness (Craig, 2009; Critchley, Wiens, Rotshtein, Ohman, \& Dolan, 2004), and adaptive human behaviors (Vinod Menon \& Uddin, 2010; Singer, Critchley, \& Preuschoff, 2009). Dysfunction of the human insula is prominent in many psychiatric and neurological disorders (Jilka et al., 2014; King-Casas et al., 2008; Sha, Wager, Mechelli, \& He, 2019). Human functional magnetic resonance imaging (fMRI) studies have demonstrated insula involvement in detection and attentional capture of goal-relevant stimuli across a wide range of cognitive tasks (Cai, Ryali, Chen, Li, \& Menon, 2014; Dosenbach, Fair, Cohen, Schlaggar, \& Petersen, 2008; Dosenbach et al., 2006; Vinod Menon \& Uddin, 2010; Sridharan, Levitin, \& Menon, 2008). Despite its critical role in cognitive function and dysfunction (Vinod Menon, 2011; Uddin, 2015), the electrophysiological foundations of the insula, its hierarchical organization, and sub-second spatiotemporal dynamics are poorly understood, part due to the low temporal resolution of fMRI data. Neuronal oscillations are a fundamental feature of cortical networks and are critical for facilitating communication among brain areas (Buzsáki \& Draguhn, 2004). Understanding the fundamental features of insula oscillations is therefore important given its rich connectivity with widespread cortical regions (Vinod Menon \& Uddin, 2010). Here we address these challenges and investigate, for the first time, the hierarchical organization and spatiotemporal gradients in the insula using a unique human intracranial electroencephalography (iEEG) dataset.

Human fMRI studies have shown that the insula plays a foundational role in human cognitive processes by interacting with other large-scale brain networks such as the medial default mode network (DMN) and lateral frontal-parietal network (FPN), which are two other large-scale brain networks that play an important role in external stimulus-related cognition and monitoring internal mental processes (V. Menon, 2011; Power et al., 2011). Crucially, the intrinsic connectivity of the insula displays close correspondence with task-related coactivation patterns (Sridharan et al., 2008; Supekar \& Menon, 2012), and this correspondence has allowed intrinsic and task-related connectivity associated with the insula to be demarcated and studied under a common, triple-network model framework (V. Menon, 2011).

Cytoarchitectonics studies in non-human primates have shown functional subdivisions within the insula. These studies have revealed that the non-human primate insula can be divided into an anterior-basal agranular part and a posterior granular part (Augustine, 1996; Mesulam \& Mufson., 1985). These studies, as well as direct tract-tracing studies, have also shown that the anterior insula (AI) is structurally connected to orbitofrontal, entorhinal, amygdala, periamygdaloid, piriform, olfactory cortices, and the temporal pole whereas the posterior insula (PI) is connected to primary and secondary sensory and motor cortices (Fudge, Breitbart, Danish, \& Pannoni, 2005; Höistad \& Barbas, 2008; Mesulam \& Mufson., 1985; Stefanacci \& Amaral, 2002). Similar to the findings in non-human primates, MRI studies in humans have also shown subdivisions in the insula (Ghaziri et al., 2017; Morel, Gallay, Baechler, Wyss, \& Gallay, 2013). Previous fMRI studies have shown functional subdivisions within the insula, as the AI was involved in task-related engagement and disengagement with the DMN and FPN across a wide range of cognitive and affective tasks (Cai et al., 2016; Cai, Ryali, Pasumarthy, Talasila, \& Menon, 2021; Chen, Cai, Ryali, Supekar, \& Menon, 2016; Lamm \& Singer, 2010; Sridharan et al., 2008). The AI, along with the anterior cingulate cortex, are known as the salience network, 
which are activated simultaneously across most cognitive task domains, thus forming a core taskset system (Dosenbach et al., 2008; Dosenbach et al., 2006; Ouyang, Li, Dang, \& Richards, 2008). Together, these findings suggest a common hierarchical organization within the insula across species.

Neuronal oscillations are a fundamental feature of cortical networks and are critical for facilitating communication among a wide-range of brain areas across species (Buzsáki \& Draguhn, 2004). Understanding the fundamental features of insula oscillations is therefore important given its rich connectivity with widespread cortical regions (Vinod Menon \& Uddin, 2010). However, the electrophysiological basis of the functional subdivisions in the insula and its spatiotemporal gradients and hierarchical organization of spontaneous neuronal oscillations are poorly understood. iEEG recordings with adequate sampling of electrodes from both the anterior and posterior subdivisions of the insula are one way to address this challenge. We examined a resting-state iEEG dataset of 10 participants, who underwent treatment for intractable epilepsy, and had a total of 239 electrodes implanted across both anterior and posterior subdivisions of the insula. The first goal of our study was to determine the hierarchical spatial organization of the electrophysiological patterns across the insula, in particular, explaining how they relate to the frequency and power of neuronal oscillations. Global brain intrinsic gradients have been known to be closely associated with the structural connectivity of brain areas and the degree of taskrelated activations (Huntenburg, Bazin, \& Margulies, 2018; Paquola et al., 2019; J. Zhang et al., 2019). Analyses of intrinsic oscillatory gradients in the insula are therefore crucial to understand the structure-function relationship of areas within the insula and between the insula and other regions of the cortex (Huntenburg et al., 2018; Vázquez-Rodríguez et al., 2019) and provide a more mechanistic understanding of oscillations as a means of intra- and inter-insula communication in the human brain. Based on recent findings that showed global-brain posteriorto-anterior frequency gradient and anterior-to-posterior power gradient using resting-state magnetoencephalography (MEG) recordings from humans (Mahjoory, Schoffelen, Keitel, \& Gross, 2020), as well as similar findings from direct recordings at the cortical surface (H. Zhang, Watrous, Patel, \& Jacobs, 2018), we hypothesized that the insula would have posterior-toanterior frequency gradient and anterior-to-posterior power gradient as well.

Recent studies in humans suggested that the hierarchical organization of neuronal activity across the cortex is not a stationary phenomenon, rather that it has a dynamic phenomenon, which propagates across the cortex in space and time (Alamia \& VanRullen, 2019; Halgren et al., 2019; Hangya et al., 2011; Kleen et al., 2021; Lozano-Soldevilla \& VanRullen, 2019; Massimini, Huber, Ferrarelli, Hill, \& Tononi, 2004; Muller et al., 2016; Stolk et al., 2019; H. Zhang \& Jacobs, 2015; H. Zhang et al., 2018). This phenomenon, also known as 'traveling waves', consist of oscillations that propagate progressively across the cortex. Traveling waves have been shown to be relevant for visual perception (Besserve, Lowe, Logothetis, Schölkopf, \& Panzeri, 2015; Davis, Muller, Martinez-Trujillo, Sejnowski, \& Reynolds, 2020; Gabriel \& Eckhorn, 2003; Muller, Reynaud, Chavane, \& Destexhe, 2014; Nauhaus, Busse, Carandini, \& Ringach, 2009; Townsend et al., 2015; Vinck et al., 2010; Zanos, Mineault, Nasiotis, Guitton, \& Pack, 2015) and movement initiation (Balasubramanian et al., 2020; Denker et al., 2018; Rubino, Robbins, \& Hatsopoulos, 2006; Rule, Vargas-Irwin, Donoghue, \& Truccolo, 2018; Takahashi et al., 2015) in non-human primates and visual processing (Liang et al., 2021; Stroh et al., 2013; Xu, Huang, Takagaki, \& Wu, 2007) and spatial navigation (Agarwal et al., 2014; Patel, Fujisawa, Berényi, 
Royer, \& Buzsáki, 2012; Patel, Schomburg, Berényi, Fujisawa, \& Buzsáki, 2013) in rodents. In humans, traveling waves have been observed during sleep (Dickey et al., 2021; Hangya et al., 2011; Massimini et al., 2004; Muller et al., 2016) as well as during behavior (Alamia \& VanRullen, 2019; Kleen et al., 2021; Stolk et al., 2019; H. Zhang \& Jacobs, 2015; H. Zhang et al., 2018). However, it is unclear whether these traveling waves, which are mostly observed on the surface of the cortex, are also relevant for deep brain structures such as the insula.

In light of this emerging literature, the second goal of our study was to detect traveling waves within the human insula. We adopted a novel method using circular statistics to identify traveling waves amidst the neuronal oscillations that we measured in each participant individually. In addition to detecting traveling waves, we also analyzed differences in the characteristics of the observed traveling waves across the participants and we measured for traveling waves at a range of frequencies. Based on findings from human electrocorticography studies that showed low frequency alpha and higher frequency beta traveling waves were weakly or uncorrelated with each other in the sensorimotor cortex (Stolk et al., 2019), we used this approach to test whether there was a link between the properties of traveling waves in the human insula between different frequencies. Our findings provide the first electrophysiological evidence for the presence of spontaneous oscillatory gradients and traveling waves in the human insula.

\section{Results}

\section{Theta frequency and power gradients in the insula}

Our first goal was to investigate the spatial properties of the frequency and power gradients in the neuronal oscillations in the insula. These analyses were inspired by recent findings showing a posterior-to-anterior fast-to-slow frequency gradient and anterior-to-posterior power gradient using resting-state MEG recordings from humans (Mahjoory et al., 2020) and hippocampal posterior-to-anterior frequency gradient using iEEG recordings in human spatial navigation (Goyal et al., 2020). We examined a dataset of subjects with iEEG recordings conducted during rest and used spectral analyses to measure the peak frequency and spectral power of the oscillations from each electrode in the insula (Methods). Individual subjects in this dataset had multiple electrodes in their insula, including contacts in both hemispheres (Figure 1). To characterize the properties of the insula oscillations in these data, for each electrode we first detected oscillations by measuring the frequency bands where power exceeded one standard deviation above the $1 / \mathrm{f}$ spectrum using the multiple oscillations detection algorithm (MODAL) (Watrous, Miller, Qasim, Fried, \& Jacobs, 2018). Theta $(\sim 6-9 \mathrm{~Hz})$ and beta $(\sim 15-32 \mathrm{~Hz})$ were the most prominent oscillations in the insula (Figure 1). In the left hemisphere, $\sim 90 \%$ of electrodes $(\mathrm{n}=107)$ showed a peak frequency in the theta range and $99 \%$ showed a peak in the beta frequency band. In the right hemisphere, $\sim 80 \%$ of electrodes $(n=95)$ had theta peaks and $\sim 81 \%$ had beta peaks. We next conducted tests of these theta and beta oscillations to evaluate their spatial and hierarchical organization.

To localize the electrode positions within the insula while accounting for inter-subject anatomical variability (Kurth, Zilles, Fox, Laird, \& Eickhoff, 2010; Naidich et al., 2004), we used a novel approach of modeling the shape of each subject's insula and then transforming each subject's electrode position to a standard Montreal Neurological Institute (MNI) coordinate 
space using an iterative, semi-automated co-registration approach (Methods). Using this pooled dataset, we then evaluated how the spectral dynamics (frequency and power) of the measured neuronal oscillations correlated with the coordinates of the electrodes within the insula (y-axis and z-axis) across all participants. The y-coordinate of each electrode represents its position along the anterior-posterior (AP) axis and the z-coordinate corresponds to the superior-inferior (SI) axis. We separately measured oscillations within the left and right hemispheres because interhemispheric differences in structure and function could affect oscillatory organization (Jakab, Molnár, Bogner, Béres, \& Berényi, 2012; Sridharan et al., 2008).

The AP axis coordinates (y-coordinates) of electrodes within the left insula predicted the peak frequency and power of the measured oscillations in the theta band (Figure 2). In the left insula, the frequency of theta was slower in the anterior insula and faster posteriorly $(t(74)=-4.09, p<$ 0.001) (Figure 2a); the amplitude of theta oscillations was higher in the left anterior insula $(t(84)$ $=2.52, p<0.05$ ) (Figure 2c). There was no indication of theta frequency $(t(74)=-0.56, p>$ $0.05)$ or theta power $(t(74)=-0.19, p>0.05)$ gradients along the SI axis of the left insula.

In the right insula, the frequencies of theta oscillations were not hierarchically organized along the AP axis $(t(57)=-0.14, p>0.05)$ or the SI axis $(t(57)=0.07, p>0.05)$ (Figure 2b). However, theta power trended towards an AP gradient, where theta power was greater in the right anterior insula $(t(57)=1.98, p=0.053)$ (Figure 2d), consistent with the results in the left hemisphere. Theta power also showed a significant SI gradient with higher amplitudes in the superior portion of the right insula $(t(57)=2.92, p<0.01)$ (Figure 2d).

\section{Beta frequency and power gradients in the insula}

In addition to theta oscillations, we also observed widespread insula oscillations in the beta band. In the left insula, beta power showed an anterior-to-posterior gradient with higher power oscillations anteriorly $(t(84)=3.07, p<0.01$ ) (Figure 3c), however, the frequency of beta rhythms were not hierarchically organized along the AP axis $(t(84)=-0.95, p>0.05)$ or the SI axis $(t(84)=-0.59, p>0.05)$ in the left insula (Figure 3a). In the right insula, beta power also showed a significant anterior-to-posterior gradient $(t(58)=2.97, p<0.01)$ (Figure 3d), with higher power oscillations being prominent in the anterior insula. Thus, beta amplitude was greater in the anterior insula across both hemispheres. However, in the right hemisphere, the frequency of insula beta rhythms showed no gradients, in neither the $\operatorname{AP}(t(58)=-0.63, p>0.05)$ nor the SI organization $(t(58)=-1.10, p>0.05)$ (Figure 3b).

These findings reveal that spontaneous theta and beta oscillations are spatially organized across the insula, especially along the AP axis. In both hemispheres, higher amplitude beta oscillations were concentrated anteriorly, whereas theta frequency and power showed opposing AP gradients in the left insula.

\section{Oscillation clusters are traveling waves in the human insula}

Having examined the spatial characteristics of the amplitude and frequencies of the insula's oscillations, we next examined the phase dynamics of these signals to test whether they were traveling waves. A prerequisite for exhibiting a traveling wave is that a spatially contiguous 
region of cortex must show an oscillation at a single frequency. Thus, as a first step to identify traveling waves in the human insula, we sought to find oscillation clusters in each participant. We defined an oscillation cluster as a spatially contiguous group of electrodes having narrowband oscillations at a given frequency band (see Methods). To identify electrode clusters, as in earlier work (H. Zhang et al., 2018), we first removed the $1 / \mathrm{f}$ background signal from the mean power spectrum of each electrode to find narrowband oscillation peaks at a particular frequency. We then used a spatial clustering algorithm to identify all the groups of contiguous electrodes that showed oscillations at the same frequency. We defined these groups of electrodes, adjacent in both frequency and space, as an oscillation cluster.

Figure 4 shows examples of oscillation clusters in the theta and beta frequency bands for the insula electrodes in participant \#3. We found 28 oscillation clusters across the 10 participants (see Figures S1-S9 for oscillation clusters from other participants). Oscillation clusters were present at both theta and beta frequencies, with 14 oscillation clusters in the theta band and 14 oscillation clusters in the beta band, in 9 out of 10 participants (participant 4 did not have any oscillation clusters). Overall, $79 \%$ of all electrodes (186 out of 239 electrodes) participated in at least one oscillation cluster.

Our next goal was to test whether the signals on these oscillation clusters behaved as traveling waves (Methods). We first informally inspected the signals from the oscillation clusters and found that the instantaneous phases of their oscillations varied systematically with electrode locations, indicating the presence of traveling waves. Figures 5a-f show an example of the systematic variation of the instantaneous phases across a subset of electrodes for both low frequency theta and higher frequency beta oscillation clusters in participant \#3. Importantly, this example shows that a single group of electrodes could exhibit traveling waves at multiple frequencies simultaneously.

We used circular statistics (Fisher, 1993) to identify traveling waves at each time-point and electrode in a given oscillation cluster by measuring the phase of the oscillations on each electrode and testing for progressive shifts between neighboring electrodes. To do this systematically, for each electrode in a given oscillation cluster, we first identified a subcluster of neighboring electrodes located within a distance of $20 \mathrm{~mm}$. Then, for each sub-cluster, we used a two-dimensional (2-D) circular-linear regression to characterize the spatial relation between the instantaneous phases of the oscillations and the positions of the electrodes (Figure 5g). Thus, this procedure models each sub-cluster's instantaneous phase distribution as a plane wave by finding the best-fitting spatial phase gradient. The fitted phase gradient provides a quantitative estimate of the speed and direction of traveling wave propagation at each time-point (Figure 5h). With this procedure, we found that the instantaneous features of traveling waves varied across time (Figures $\mathbf{5 h}-\mathbf{j}, \mathbf{6})$. We computed the instantaneous wave-strength of the traveling waves for the given sub-cluster as the mean proportion of phase variation explained by the circular-linear models across the cluster (Methods).

To assess the statistical reliability of each cluster's traveling waves, we used a permutation procedure where we compared each cluster's average wave-strength to the distribution of wavestrength values expected by chance (Methods). This statistical approach revealed that traveling waves were reliably present in this resting-state dataset. Overall, traveling waves were present 
$\sim 35 \%$ of time across all clusters. Individual clusters varied in the prevalence of traveling waves, ranging from $\sim 10 \%$ to $\sim 87 \%$. 93\% of all insular oscillation clusters (26 out of 28 oscillation clusters) showed traveling waves at statistically significant levels. Overall, traveling waves had a median propagation speed of $\sim 0.7 \mathrm{~m} / \mathrm{s}$, which is consistent with earlier work on traveling waves across the cortical surface (H. Zhang et al., 2018).

\section{Amplitude of oscillations is positively correlated with the strength of traveling waves}

We next sought to investigate the relationship between the amplitude of the oscillations measured at individual electrodes and the strength of cluster-wide traveling waves, based on emerging findings from motor cortical local field potentials (LFPs) in non-human primates (Denker et al., 2018). Visual inspection revealed that such a link might be present, as we saw that the average amplitude of electrodes from a cluster correlated with the wave-strength of simultaneous traveling waves (Figures 7a-f).

To systematically examine how local oscillation features correlated with traveling waves, we used the Hilbert transform to measure the amplitude of the oscillations on each individual electrode and then measured the average amplitude across electrodes for each cluster at each timepoint. Then, for each cluster, we computed the correlation between mean oscillation amplitude and traveling wave-strength. We used a permutation procedure to test statistical significance (Methods). This analysis revealed that for $\sim 71 \%$ of clusters (20 out of 28 clusters), there was a statistically significant correlation between amplitude and wave-strength (Figures 7g-i, Table S1). This indicates that the strength of the traveling waves correlates with the amplitude of neuronal oscillations in the human insula.

Visual inspection also revealed temporal structure in this pattern, in which the amplitude of oscillations in a cluster peaked before wave-strength (Figures $\mathbf{7 b - c}$ ). To quantify the temporal delay between the amplitude and wave-strength time-series, we carried out a cross-correlation analysis (Rabiner \& Gold, 1975). This analysis revealed that oscillation amplitude increases prior to increases in wave-strength, with a mean shift of $\sim 8 \mathrm{~ms}$ for theta traveling waves and $\sim 4 \mathrm{~ms}$ for beta traveling waves. Together, these results suggest a putative causal role of higher amplitude oscillations underlying higher wave-strength traveling waves in the human insula.

\section{Theta and beta traveling waves are temporally uncorrelated with each other}

Finally, we investigated the relationship between the low frequency theta and higher frequency beta traveling waves, based on emerging findings that showed that low frequency alpha and higher frequency beta traveling waves are un- or weakly correlated with each other in the sensorimotor cortex (Stolk et al., 2019). To explore the relationship between the theta and beta traveling waves in our dataset, we carried out correlation analyses examining the interrelations between the instantaneous strength, phase, and oscillatory power of traveling waves between low and high frequencies, for any oscillation clusters that showed significant traveling wave clusters at multiple frequencies. To test the statistical significance of links between the features of low and high frequency traveling waves, we circularly shuffled the values of the higher and lower frequency traveling waves relative to each other to build a surrogate distribution of correlation values corresponding to the null hypothesis of the two traveling waves being unrelated. 
Overall, this analysis showed that overwhelmingly, when individual electrodes participated in multiple traveling waves, these effects were largely independent. Across all clusters that showed beta and theta traveling waves, the instantaneous strengths of these traveling waves were uncorrelated (Pearson $r, p>0.05$ ) for all but two clusters (Table S2). Beyond testing for links in wave-strength, to test for more fine-grained links between different traveling waves, we compared how instantaneous phase and power of slower theta-band traveling waves related to the strength of the beta traveling waves, however we found no such links (Pearson $r, p$ 's $>0.05$ ) (Table S2). Together, these results suggest that across the insula, low frequency theta and higher frequency beta traveling waves operate independently.

\section{Discussion}

Here we used a unique resting-state iEEG dataset of 10 participants, who underwent treatment for intractable epilepsy, and electrodes sampled along the extent of the insula with implants in both the anterior and posterior subdivisions of the insula across participants. With this dataset, we carried out a comprehensive analysis of frequency and power gradients along the oscillations we measured along the AP and SI axes in the insula, for the first time. Using spectral analyses, we found that oscillations in the theta and beta bands are the most prominent spontaneous oscillations in the insula (Figure 1), and these oscillations are largely organized along the AP axis. The left and right insula showed convergent patterns of anterior-to-posterior spectral power gradient in the beta band ( $\sim 15-32 \mathrm{~Hz})$. Intriguingly, theta $(\sim 6-9 \mathrm{~Hz})$ oscillations were heterogeneous across hemispheres and showed a gradient of frequency that matched the insula's hierarchical organization. In the left insula, theta oscillations showed a posterior-to-anterior frequency gradient and anterior-to-posterior power gradient. However, in the right insula, theta oscillations showed a superior-to-inferior power gradient, but no frequency gradient.

We next identified traveling waves corresponding to the theta and beta oscillations. We identified spatially contiguous clusters of electrodes at theta and beta frequency bands and then used a novel, localized circular-linear regression approach to detect traveling waves, assuming that the relative phases of the oscillation clusters exhibit a linear relationship with electrode locations locally. In addition to detecting traveling waves in most of these participants, we also found that the amplitudes of neuronal oscillations are positively correlated with the strength of traveling waves in the insula. Furthermore, this analysis also revealed that theta and beta oscillatory traveling waves in the insula are uncorrelated with each other. These findings provide a more complete understanding of spontaneous neuronal oscillations in the human insula by showing that this structure shows multiple independent traveling waves during rest.

The insula is a key brain region which plays a crucial role in a wide range of adaptive human behaviors explored using fMRI (Vinod Menon \& Uddin, 2010; Singer et al., 2009), however, its electrophysiological foundations has remained unexplored due to the lack of sufficient electrode coverage. Recently, with the increased popularity of stereo EEG recording technology (Abou-AlShaar, Brock, Kundu, Englot, \& Rolston, 2018), we have much more access to deep brain structures such as the insula in epilepsy patients and can explore the electrophysiological foundations, sub-second dynamics, and spatiotemporal gradients in this area for the first time. Crucially, our findings of spontaneous gradients and traveling waves in the insula suggest a 
fundamental new principle that could be used by the insula to link its processing to widespread cortical areas.

Hierarchical organization of spectral frequency and power of theta and beta oscillations in the human insula

The first goal of our study was to detect frequency and power gradients in the human insula. Our analysis of hierarchical organization of theta oscillations in the insula revealed that, in the left insula, theta oscillations have a posterior-to-anterior frequency gradient and anterior-toposterior power gradient. This finding is consistent with global-brain posterior-to-anterior frequency gradient and anterior-to-posterior power gradient in resting-state MEG recordings from humans (Mahjoory et al., 2020) as well human iEEG recordings (H. Zhang et al., 2018) and hippocampal posterior-to-anterior frequency gradient in iEEG recordings in humans performing virtual spatial navigation tasks (Goyal et al., 2020). Our findings show that the frequency and power gradients observed in other parts of the cortical areas also extend to the insula. Our findings also corroborate previous resting-state fMRI (Deen, Pitskel, \& Pelphrey, 2011; Faillenot, Heckemann, Frot, \& Hammers, 2017) and diffusion MRI (V. Menon et al., 2020) findings in humans which have identified distinct functional subdivisions in the insula based on differential patterns of intrinsic functional connectivity and extend them to electrophysiological data. Findings also converge on the presence of spindle-shaped von Economo neurons in AI (Cobos \& Seeley, 2015; Evrard, Forro, \& Logothetis, 2012; Seeley et al., 2012; von Economo, 1926; Watson, Jones, \& Allman, 2006), which form the agranular part of the insula. The AI lacks external (layer II) and internal granular (IV) layers, which may subserve its special role of integrating information across wide-spread neuronal networks (Mesulam \& Mufson., 1985; Morel et al., 2013; Wylie et al., 2015). By showing that there are distinctive patterns of oscillations for the anterior versus posterior insula that match broader cortex-wide patterns, it supports the notion that the insula is a communication hub and suggests that distinctive patterns of oscillations could play a role in allowing it to engage with other regions, such as the DMN and FPN, across a wide range of cognitive and affective tasks (Cai et al., 2016; Cai et al., 2021; Chen et al., 2016; Lamm \& Singer, 2010; Sridharan et al., 2008).

In our previous study involving the AI using resting-state $\mathrm{EEEG}$ recordings in humans, we had shown that low frequency delta $(\sim 0.5-4 \mathrm{~Hz})$ oscillations underlie higher directed causal information flow from the AI to the other brain regions such as the DMN and the FPN (Das \& Menon, 2020). Crucially, this finding was replicated across multiple iEEG cohorts. Taken together, lower frequency and higher power oscillations in the AI compared to PI seems to be a putative mechanism that enables the AI to maintain stability and flexibility with other large-scale brain networks (Cai et al., 2016; Cai et al., 2021; Chen et al., 2016; Lamm \& Singer, 2010; Sridharan et al., 2008).

However, surprisingly, in the right insula, we found no AP theta gradients in frequency and only weak AP theta gradients in power. Moreover, right insula theta oscillations showed a superior-toinferior power gradient, but no frequency gradient. This finding is consistent with structural MRI studies in humans which have shown a left insula dominated connectivity profile with the prefrontal and frontal brain regions (Jakab et al., 2012). This left insula dominance has also been confirmed by other structural MRI studies (Chiarello, Vazquez, Felton, \& Leonard, 2013; Van 
Essen, Glasser, Dierker, Harwell, \& Coalson, 2012). Thus, our findings on the left insula dominated theta oscillation frequency and power gradients converge on the structural MRI studies and provide novel evidence for the electrophysiological basis of this structural asymmetry. These structural MRI studies have further shown that the anterior, rather than the posterior, insula in the left hemisphere is more involved for semantic decisions (Chiarello et al., 2013), consistent with the frequency and power gradients that we found along the AP axis of the insula. We hypothesize that these spontaneous frequency and power gradients in the left insula may also play a key role in modulating human behavior and together, with the structural MRI findings, suggest that these gradients may help the AI in integrating information from other parts of the insula and projecting them to other parts of the cortex for further processing given its connectivity with widespread cortical regions. Future studies with denser sampling of electrodes in both the anterior and posterior parts of the insula are needed to further probe the hemispheric heterogeneity of low frequency gradients observed in our analyses.

We next investigated the hierarchical organization of beta oscillations. This analysis revealed that both the left and right insula have anterior-to-posterior spectral power gradient. Gradients in the human brain in previous iEEG studies have predominantly been found in low frequency oscillations (Das \& Menon, 2020; Goyal et al., 2020; H. Zhang et al., 2018). However, consistent with our results, some recent electrophysiological studies have also found a crucial role for beta oscillations in feedback signaling in both non-human primate and human brains (Bastos et al., 2015; Das \& Menon, 2021). Beta oscillations have been hypothesized to underlie directed causal information flow from one brain region to another and may contribute to transitioning latent neuronal ensembles into "active" representations (Spitzer \& Haegens, 2017) as well as the subsequent maintenance of information in cell assemblies (Engel \& Fries, 2010). Thus, higher beta power in the AI facilitates communication with other large-scale brain networks via feedback signaling. In summary, the frequency gradients were most prominent in the left insula whereas the power gradients were present in both hemispheres. We hypothesize that the left hemisphere insula communicates via both frequency and power gradients whereas the right hemisphere insula communicates via power gradients. Multiplexing of frequency and power gradients and hemispheric asymmetry may help the AI maintain a powerful and flexible role in cognitive control mechanisms as hypothesized previously in human fMRI studies (Cai et al., 2016; Cai et al., 2021; Chen et al., 2016; Lamm \& Singer, 2010; Sridharan et al., 2008).

\section{Oscillation clusters in the human insula are spatially localized traveling waves}

Our second goal was to detect traveling waves of theta and beta frequency oscillations. We found that traveling waves are present $\sim 35 \%$ of time in the human insula across all clusters, indicating that traveling waves are relatively sparse across participants. Our findings converge with restingstate rodent (Matsui, Murakami, \& Ohki, 2016) and human (Bahramisharif et al., 2013; Halgren et al., 2019) electrophysiology studies as well as more recent resting-state human fMRI studies (Raut et al., 2021) which have detected traveling waves of spontaneous neuronal oscillations and extend these findings to the human insula. These findings also converge on recent findings which have detected relatively sparse traveling waves in the rodent somatosensory cortex (Moldakarimov, Bazhenov, Feldman, \& Sejnowski, 2018), visual cortex of non-human primates (Davis et al., 2020), and iEEG recordings in humans (H. Zhang et al., 2018). Intriguingly, previous resting-state iEEG studies in humans (Bahramisharif et al., 2013; Halgren et al., 2019) 
have found traveling waves in the low frequency alpha oscillations only. However, the role of traveling waves of spontaneous beta oscillations in the human cortex is relatively unknown, although traveling waves have been detected during sleep spindles, which tend to occur in the beta frequency range $(\sim 11-15 \mathrm{~Hz})$ (Muller et al., 2016). Our finding of traveling waves in the beta frequency band in the human insula suggest a prominent role for beta band for communicating information within the insula and also dynamically coordinating this information with other brain networks and may explain the role of insula in a wide-range of adaptive human behaviors (Vinod Menon \& Uddin, 2010), which has been hypothesized as an important role of beta frequency oscillations (Betti, Della Penna, de Pasquale, \& Corbetta, 2021).

Lack of sufficient electrode placements (Figures S1-S9) simultaneously in both the anteriorposterior and dorsal-ventral portions of the insula in individual participants precluded population-level analysis of the propagation directions of the traveling waves. Nevertheless, analysis of the propagation directions of the traveling waves in select participants with adequate electrode sampling (Figures 5-6) revealed directional modulation of insular traveling waves, however they were different across participants. Future studies with adequate sampling of electrodes in the insula of individual participants are needed to rigorously analyze the instantaneous propagation directions of traveling waves and relate them directly to the frequency and power gradients that we observed in the insula.

\section{Features of theta and beta traveling waves in the human insula}

We next investigated the relationship between the amplitude of low and high frequency oscillations and the strength of traveling waves for the oscillation clusters. For $\sim 71 \%$ of clusters, we found a statistically significant correlation between amplitude and wave-strength and for 19 out of these 20 clusters, we found a positive correlation between amplitude and wave-strength. Cross-correlation analysis revealed that the median temporal delay between amplitude and wavestrength is $\sim 8 \mathrm{~ms}$ for theta traveling waves and $\sim 4 \mathrm{~ms}$ for beta traveling waves, with the wavestrength time-series leading the amplitude time-series in both cases. Together, these time shifts suggest a putative causal role of higher amplitude oscillations underlying higher wave-strength traveling waves in the human insula. Finally, we investigated the relationship between traveling waves at theta and beta frequencies. Recent findings in human electrocorticography studies have shown that low frequency alpha and higher frequency beta traveling waves are temporally uncorrelated or weakly correlated with each other in the sensorimotor cortex (Stolk et al., 2019). Our analysis confirmed that this pattern also persists in the human insula.

Interestingly, our findings also converge on a recent study (Marks et al., 2021) which used a large dataset of iEEG recordings from 164 participants showing temporal independence of low and high frequency oscillations during the memory encoding period of a verbal episodic memory task. Temporal uncorrelatedness of theta and beta traveling waves in the human insula suggests putative orthogonal frequency channels of spatiotemporal information transfer within the insula. We hypothesize that these spatiotemporally orthogonal channels enable the insula to operate at the apex of cognitive control networks observed in the human brain (Cai et al., 2021) and initiate dynamic switching, with the DMN and FPN, which has been reported across a wide range of cognitive and affective tasks (Cai et al., 2016; Cai et al., 2021; Chen et al., 2016; Lamm \& Singer, 2010; Sridharan et al., 2008). 


\section{Conclusions}

The insula plays a critical role in a wide range of adaptive human behaviors (Vinod Menon \& Uddin, 2010; Singer et al., 2009) and insula dysfunction is prominent in many psychiatric and neurological disorders (Jilka et al., 2014; King-Casas et al., 2008; Sha et al., 2019). Crucially, the intrinsic connectivity of the insula displays close correspondence with task-related coactivation patterns, and this correspondence has allowed the intrinsic and task-related connectivity associated with the insula to be demarcated and studied under a common framework (Sridharan et al., 2008; Supekar \& Menon, 2012). Using a unique dataset of resting-state human iEEG recordings from 10 participants and 239 electrodes in the insula, we probed its hierarchical organization and spatiotemporal dynamics, and for the first time, detected traveling waves of spontaneous neuronal oscillations in theta and beta frequency bands in the human insula. Our findings significantly advance the understanding of the neurophysiological basis of spontaneous neuronal oscillations in the insula, and more broadly, foundational mechanisms underlying largescale cortical hierarchy in the human brain. Crucially, our findings of spontaneous gradients and traveling waves in the insula suggest a fundamental new principle that could be used by the insula to link its processing to widespread cortical areas. 


\section{Methods}

\section{Participants}

Intracranial electroencephalography (iEEG) recordings were acquired from 10 patients $(5$ females; 9 right-handed, 1 ambidextrous) with drug-intractable epilepsy. The average age of the patients was $\sim 31$ with an age of seizure onset between 9 months old and 46 years old. All decisions regarding the location and coverage of the iEEG probes were based solely on clinical criteria. The Baylor College of Medicine Institutional Review Board approved placement of all electrodes (IRB-18112). All patients provided informed consent before participating. The insula was not the origin of seizure onset for any patients in this study.

\section{Electrophysiological recordings and preprocessing}

We used resting state electrophysiological recordings from participants performing a passive fixation resting state task, where they focused on a white crosshair at the center of a black background on a computer monitor for $\sim 5$ minutes duration. Data was recorded from 239 iEEG electrodes using a neural signal processor (Blackrock Microsystems). The spacing between neighboring electrodes varied from $3.5 \mathrm{~mm}$ to $5.53 \mathrm{~mm}$ (center-to-center). The data was amplified, band-pass filtered $(0.3 \mathrm{~Hz}-7.5 \mathrm{kHz})$, and digitized at $2 \mathrm{kHz}$. For analysis of spectral frequency and power gradients, we used bipolar referencing (iEEG electrodes re-referenced to their closest neighbors) and for analysis of traveling waves, we used common average referencing (iEEG electrodes re-referenced to the average signal of all electrodes). Automatic data rejection was performed with EEGLAB based on $3 \mathrm{x}$ the standard deviation of spectra from $1-50 \mathrm{~Hz}$ in order to remove data with artifactual voltage deflections and epileptiform discharges (Delorme \& Makeig, 2004). Each subject contributed an average of $23.9( \pm 2.43)$ electrodes, with $12.5( \pm 4.04)$ in the left insula and $11.4( \pm 2.55)$ in the right insula. Four of the ten subjects had bilateral insula coverage. A zero-phase notch filter was used to remove $60 \mathrm{~Hz}$ line noise and harmonics.

\section{Electrode localization}

An automatic cortical reconstruction was performed on the preoperative T1-weighted MRI FreeSurfer (http://surfer.nmr.mgh.harvard.edu/). All iEEG electrodes were localized by coregistering each patients' pre-surgical structural T1-weighted MRI with their post-surgical CT images (Fischl, 2012) using the Functional Magnetic Resonance Imaging for the Brain Software Library's (FMRIB's) Linear Image Registration Tool (FLIRT) (Jenkinson, Bannister, Brady, \& Smith, 2002; Jenkinson \& Smith, 2001). All neuroimages were co-registered to standard MNI 152 space in an iterative semi-automated manner. With ITK-SNAP (version 3.8.0) (Yushkevich et al., 2006), the pre-surgical T1-weighted MRI was manually aligned to the standard MNI 152 atlas image before a rigid registration was performed. A global affine transformation was subsequently applied to refine the co-registration. Bilateral insula regions from the MNI 152 atlas were dilated by 5 voxels and combined to create a mask, which was then used in the final registration step. A local affine transformation with this mask was applied three times to optimize insular co-registration from patient space to MNI 152 space. Mutual information was used as the similarity metric for all registrations. The accuracy of each registration was manually 
verified before the resulting transformation matrix was applied to the post-surgical CT images. With the post-surgical CT registered to MNI 152 space, the resulting electrode coordinates were localized and extracted using BioImage Suite (Joshi et al., 2011). Electrodes located in the insula were first visually identified by a neurosurgeon and further verified using the latest DesikanKilliany (DK) atlas, available in the Freesurfer software (Desikan et al., 2006; Fischl, 2012). Anatomical parcellation of the insula for image generation was performed using the DK atlas.

\section{Analysis of frequency and power gradients}

Power spectral density (PSD) was computed for each electrode via wavelet convolution. Each signal, $x(t)$, from a given electrode, was convolved with a Morlet wavelet defined by $\omega(t, f)=$ $A * e^{\frac{-t^{2}}{2 \sigma_{t}^{2}}} * e^{i 2 \pi f_{t}}$, where $t$ is time, $f$ is frequency, and $\sigma_{t}$ is wavelet duration. $A$ is normalized amplitude, such that $A=\sigma_{t} \sqrt{\pi}^{-1 / 2}$. The number of cycles in each wavelet function was 6 . Frequency bands for spatial gradient analyses were selected from the average of all electrodes using an oscillation detecting procedure called the multiple oscillations detection algorithm (MODAL) (Watrous et al., 2018). MODAL detects frequency bands where power exceeds one standard deviation above the $1 / \mathrm{f}$ background spectrum. Across the insula, theta $(\sim 6-9 \mathrm{~Hz})$ and beta $(\sim 15-32 \mathrm{~Hz})$ oscillations were detected (Figure 1). Peak frequencies and the corresponding power within theta and beta bands were selected by applying a robust (bisquare) linear fit to the power spectral density function and then locating the frequencies that corresponded to the maximum amplitude above the residuals.

\section{Traveling waves analysis}

We adopted a novel method to identify traveling waves in the insula corresponding to neuronal oscillations in each participant individually. These consisted of two primary steps: (i) identification of spatially contiguous clusters of electrodes at theta and beta bands, and (ii) identification of systematic spatial variation of instantaneous phases of the electrodes for each cluster, defined to be a traveling phase wave. These are detailed below.

\section{Identification of spatial clusters}

To characterize propagating traveling waves, we first sought to identify spatial clusters of electrodes with narrowband oscillations at theta and beta bands. We adopted methods similar to our previous approach (H. Zhang et al., 2018). An advantage of this algorithm was that it accounted for several complexities of human brain oscillations measured with iEEG signals, including differences in electrode positions across subjects and variations in oscillation frequencies across individuals.

Similar to the PSD analysis above, we first used Morlet wavelets to compute the power of the neuronal oscillations throughout the resting-state recordings of approximately five minutes duration at 200 frequencies logarithmically spaced from 3 to $40 \mathrm{~Hz}$. To identify narrowband oscillations at each site, we fit a line to each patient's mean power spectrum in log-log coordinates using a robust linear regression as above (Figure 4). We then subtracted the actual power spectrum from the regression line. This normalized power spectrum removes the $1 / \mathrm{f}$ 
background signal and emphasizes narrowband oscillations as positive deflections. We identified narrowband peaks in the normalized power spectrum as any local maximum greater than one standard deviation above the mean.

Next, we implemented a spatial clustering algorithm to identify oscillation clusters, which we defined as contiguous groups of electrodes in each subject that exhibited narrowband oscillations at theta $(\sim 4-8 \mathrm{~Hz})$ and beta $(\sim 12-30 \mathrm{~Hz})$ frequencies. To test whether the electrodes comprised a spatially contiguous group, we created a pairwise-adjacency matrix to judge their spatial proximity. This matrix indicated whether each electrode pair was separated by less than 20 Talairach units $(20 \mathrm{~mm})$. Finally, we used this adjacency matrix to identify mutually connected spatial clusters of electrodes by computing the connected components of this graph (Tarjan, 1972). We included in our analyses only clusters with at least four connected electrodes.

\section{Identification of traveling waves}

We next identified traveling waves corresponding to the spatially contiguous groups of electrodes oscillating at theta and beta frequencies (Figures 4, S1-S9). A traveling wave can be described as an oscillation that moves progressively across a region of cortex. Quantitatively, a traveling phase wave can be defined as a set of simultaneously recorded neuronal oscillations at the same frequency band whose instantaneous phases vary systematically with the location of the recording electrode. We used a localized circular-linear regression approach, assuming that the relative phases of the oscillation clusters exhibit a linear relationship with electrode locations locally. This locally circular-linear fitting of phase-location can detect complex patterns (Ermentrout \& Kleinfeld, 2001; Muller et al., 2016) of traveling waves in an oscillation cluster in addition to linear traveling waves. This is different than our previous approach $(\mathrm{H}$. Zhang et al., 2018) in which we carried out circular-linear fitting of phase-location for an entire oscillation cluster assuming that instantaneous relative phases exhibit a linear relationship with electrode location for the entire oscillation cluster.

To identify traveling waves from the phases of each oscillation cluster, we first measured the instantaneous phases of the signals from each electrode of a given cluster by applying a $3^{\text {rd }}$ order Butterworth filter at the cluster's narrowband peak frequency (bandwidth $\left[\mathrm{f}_{\mathrm{p}} \times .85, \mathrm{f}_{\mathrm{p}} / .85\right]$ where $\mathrm{f}_{\mathrm{p}}$ is the peak frequency). We used Hilbert transform on each electrode's filtered signal to extract the instantaneous phases.

We used circular statistics to identify traveling waves of phase progression for each oscillation cluster at each time point (Fisher, 1993). For each spatial phase distribution, we used twodimensional (2-D) localized circular-linear regression to assess whether the observed phase pattern varied linearly with the electrode's coordinates in 2-D. In this regression, for each electrode in a given oscillation cluster, we first identified the neighboring electrodes that were located within $20 \mathrm{~mm}$ distance of the given electrode, constituting a sub-cluster of the given cluster. We included in our subsequent analyses only sub-clusters with at least four electrodes. Let $x_{i}$ and $y_{i}$ represent the 2-D coordinates and $\theta_{i}$ the instantaneous phase of the $i$ th electrode. The 2-D coordinates $x_{i}$ and $y_{i}$ are determined by projecting the 3-D Talairach coordinates for each sub-cluster into the best-fitting 2-D plane using the principal component analysis. We projected the electrode coordinates into a 2-D space to simplify visualizing and interpreting the data. 
We used a 2-D circular-linear model

$$
\hat{\theta}_{i}=\left(a x_{i}+b y_{i}+\vartheta\right) \bmod 360^{0}
$$

where $\hat{\theta}_{i}$ is the predicted phase, $a$ and $b$ are the phase slopes corresponding to the rate of phase change (or spatial frequencies) in each dimension, and $\vartheta$ is the phase offset. We converted this model to polar coordinates to simplify fitting. We define $\alpha=\operatorname{atan} 2(b, a)$ which denotes the angle of wave propagation and $\xi=\sqrt{a^{2}+b^{2}}$ which denotes the spatial frequency. Circular-linear models do not have an analytical solution and must be fitted iteratively (Fisher, 1993). We fitted $\alpha$ and $\xi$ to the distribution of oscillation phases at each time point by conducting a grid search over $\alpha \in\left[0^{0}, 360^{\circ}\right]$ and $\xi \in[0,32.5]$ in increments of $5^{0}$ and $0.5^{\circ} / \mathrm{mm}$, respectively (Note that $\xi=$ 32.5 corresponds to the spatial Nyquist frequency of $32.5 \% \mathrm{~mm}$ corresponding to the highest spacing between neighboring electrodes of $5.53 \mathrm{~mm}$.). The model parameters $(a=\xi \cos (\alpha)$ and $b=\xi \sin (\alpha)$ ) for each time point are fitted to most closely match the phase observed at each electrode in the sub-cluster. We computed the goodness of fit as the mean vector length $\bar{r}$ of the residuals between the predicted $\left(\hat{\theta}_{i}\right)$ and actual $\left(\theta_{i}\right)$ phases (Fisher, 1993),

$$
\bar{r}=\sqrt{\left[\frac{1}{n} \sum_{i=1}^{n} \cos \left(\theta_{i}-\hat{\theta}_{i}\right)\right]^{2}+\left[\frac{1}{n} \sum_{i=1}^{n} \sin \left(\theta_{i}-\hat{\theta}_{i}\right)\right]^{2}},
$$

where $n$ is the number of electrodes. The selected values of $\alpha$ and $\xi$ are chosen to maximize $\bar{r}$. This procedure is repeated for each sub-cluster of a given oscillation cluster. To measure the statistical reliability of each fitted traveling wave, we examined the phase variance that was explained by the best fitting model. To do this, we computed the circular correlation $\rho_{c c}$ between the predicted $\left(\hat{\theta}_{i}\right)$ and actual $\left(\theta_{i}\right)$ phases at each electrode:

$$
\rho_{c c}=\frac{\sum_{i=1}^{n} \sin \left(\theta_{i}-\bar{\theta}\right) \sin \left(\hat{\theta}_{i}-\overline{\hat{\theta}}\right)}{\sqrt{\sum_{i=1}^{n} \sin ^{2}\left(\theta_{i}-\bar{\theta}\right) \sum_{i=1}^{n} \sin ^{2}\left(\hat{\theta}_{i}-\overline{\hat{\theta}}\right)}},
$$

where bar denotes averaging across electrodes. Finally, we applied an adjustment for number of fitted model parameters:

$$
\rho_{a d j}^{2}=1-\frac{\left(1-\rho_{c c}^{2}\right)(n-1)}{n-k-1},
$$

where $k$ is the number of independent regressors ( $k=3$ in this case). We refer to $\rho_{a d j}^{2}$ as the wavestrength of the traveling wave (H. Zhang et al., 2018) as it quantifies the strength of the traveling 
wave (note that $\rho_{a d j}^{2}$ has been referred to as phase gradient directionality (PGD) in some prior studies (Muller et al., 2016; Rubino et al., 2006; H. Zhang et al., 2018)).

\section{Statistical analysis}

We used linear mixed-effects models to test the statistical significance of the frequency and power gradients. For analysis of frequency gradients, the peak frequency of each electrode was the dependent variable and the y- (AP) and z- (SI) coordinates of the electrodes were used as independent variables. Furthermore, a categorical independent variable, indicating the subject that contributed to the given frequency, was also included to account for variance across subjects. Interaction terms between all independent variables were included in the models as well. The spatial models were constructed as follows, EPHYS $=\beta_{0}+\beta_{A P}+\beta_{S I}+\beta_{S U B J}+$ $\left(\beta_{A P} * \beta_{S I}\right)+\left(\beta_{A P} * \beta_{S U B J}\right)+\left(\beta_{S I} * \beta_{S U B J}\right)$, where EPHYS can represent spectral frequency, or spectral power. Left and right hemisphere data were analyzed separately because they are known to play different functional roles that may contribute to differences in oscillatory organization (Sridharan et al., 2008). False discovery rate (FDR) correction was applied to all resulting pvalues to control for multiple comparisons.

Surrogate analysis was used to test statistical significance of the detected traveling waves. The coordinates of the electrodes with respect to the Hilbert-transformed phases were shuffled so that the contiguous spatial variation of the phases is destroyed. This ensured that our statistical test specifically evaluated the spatial arrangement of the recording electrodes. For each iteration of this procedure, the circular-linear regression analysis was repeated on this shuffled data to build a distribution of surrogate $\rho_{a d j}^{2}$ values against which the observed $\rho_{a d j}^{2}$ was tested $(p<0.05)$. Similarly, to test the statistical significance of the correlations for amplitude- $\rho_{a d j}^{2}, \rho_{a d j}^{2}($ low $)$ $\rho_{a d j}^{2}$ (high), phase (low)- $\rho_{a d j}^{2}$ (high), and power (low)- $\rho_{a d j}^{2}$ (high), the values from one time-series were circularly shuffled with respect to the other so that the instantaneous correlation between the two time-series is destroyed. We used standard Pearson correlation coefficients to test the correlations for amplitude- $\rho_{a d j}^{2}, \rho_{a d j}^{2}$ (low)- $\rho_{a d j}^{2}$ (high), and power (low)- $\rho_{a d j}^{2}$ (high). We used circular-linear correlation coefficients (Johnson \& Wehrly, 2020; Mardia, 2020) to compare the correlations involving phase (i.e., phase (low)- $\rho_{a d j}^{2}$ (high)).

\section{Acknowledgements}

We thank Drs. Uma Mohan and Honghui Zhang for help in traveling waves analysis. This research was supported by NIH grants R01-MH127006 and K01-MH116364 to K.B. and B.M., an NSF CAREER award to J.J., and grants from McNair Foundation and Dana Foundation to S.A.S. 


\section{References}

Abou-Al-Shaar, H., Brock, A. A., Kundu, B., Englot, D. J., \& Rolston, J. D. (2018). Increased nationwide use of stereoencephalography for intracranial epilepsy electroencephalography recordings. J Clin Neurosci, 53, 132-134. doi:10.1016/j.jocn.2018.04.064

Agarwal, G., Stevenson, I. H., Berényi, A., Mizuseki, K., Buzsáki, G., \& Sommer, F. T. (2014). Spatially distributed local fields in the hippocampus encode rat position. Science, 344(6184), 626-630. doi:10.1126/science.1250444

Alamia, A., \& VanRullen, R. (2019). Alpha oscillations and traveling waves: Signatures of predictive coding? PLoS Biol, 17(10), e3000487. doi:10.1371/journal.pbio.3000487

Augustine, J. R. (1996). Circuitry and functional aspects of the insular lobe in primates including humans. Brain Res Brain Res Rev, 22(3), 229-244. doi:10.1016/s0165-0173(96)00011-2

Bahramisharif, A., van Gerven, M. A., Aarnoutse, E. J., Mercier, M. R., Schwartz, T. H., Foxe, J. J., . . Jensen, O. (2013). Propagating neocortical gamma bursts are coordinated by traveling alpha waves. J Neurosci, 33(48), 18849-18854. doi:10.1523/jneurosci.245513.2013

Balasubramanian, K., Papadourakis, V., Liang, W., Takahashi, K., Best, M. D., Suminski, A. J., \& Hatsopoulos, N. G. (2020). Propagating Motor Cortical Dynamics Facilitate Movement Initiation. Neuron, 106(3), 526-536.e524. doi:10.1016/j.neuron.2020.02.011

Bastos, A. M., Vezoli, J., Bosman, C. A., Schoffelen, J. M., Oostenveld, R., Dowdall, J. R., .. . Fries, P. (2015). Visual areas exert feedforward and feedback influences through distinct frequency channels. Neuron, 85(2), 390-401. doi:10.1016/j.neuron.2014.12.018

Besserve, M., Lowe, S. C., Logothetis, N. K., Schölkopf, B., \& Panzeri, S. (2015). Shifts of Gamma Phase across Primary Visual Cortical Sites Reflect Dynamic StimulusModulated Information Transfer. PLoS Biol, 13(9), e1002257. doi:10.1371/journal.pbio.1002257

Betti, V., Della Penna, S., de Pasquale, F., \& Corbetta, M. (2021). Spontaneous Beta Band Rhythms in the Predictive Coding of Natural Stimuli. Neuroscientist, 27(2), 184-201. doi:10.1177/1073858420928988

Buzsáki, G., \& Draguhn, A. (2004). Neuronal oscillations in cortical networks. Science, 304(5679), 1926-1929. doi:10.1126/science.1099745

Cai, W., Chen, T., Ryali, S., Kochalka, J., Li, C. S., \& Menon, V. (2016). Causal Interactions Within a Frontal-Cingulate-Parietal Network During Cognitive Control: Convergent Evidence from a Multisite-Multitask Investigation. Cereb Cortex, 26(5), 2140-2153. doi:10.1093/cercor/bhv046

Cai, W., Ryali, S., Chen, T., Li, C. S., \& Menon, V. (2014). Dissociable roles of right inferior frontal cortex and anterior insula in inhibitory control: evidence from intrinsic and taskrelated functional parcellation, connectivity, and response profile analyses across multiple datasets. J Neurosci, 34(44), 14652-14667. doi:10.1523/jneurosci.3048-14.2014

Cai, W., Ryali, S., Pasumarthy, R., Talasila, V., \& Menon, V. (2021). Dynamic causal brain circuits during working memory and their functional controllability. Nat Commun, 12(1), 3314. doi:10.1038/s41467-021-23509-x

Chen, T., Cai, W., Ryali, S., Supekar, K., \& Menon, V. (2016). Distinct Global Brain Dynamics and Spatiotemporal Organization of the Salience Network. PLOS Biology, 14(6), e1002469. doi:10.1371/journal.pbio.1002469 
Chiarello, C., Vazquez, D., Felton, A., \& Leonard, C. M. (2013). Structural asymmetry of anterior insula: behavioral correlates and individual differences. Brain Lang, 126(2), 109122. doi:10.1016/j.band1.2013.03.005

Cobos, I., \& Seeley, W. W. (2015). Human von Economo neurons express transcription factors associated with Layer V subcerebral projection neurons. Cereb Cortex, 25(1), 213-220. doi:10.1093/cercor/bht219

Craig, A. D. (2009). How do you feel--now? The anterior insula and human awareness. Nat Rev Neurosci, 10(1), 59-70. doi:10.1038/nrn2555

Critchley, H. D., Wiens, S., Rotshtein, P., Ohman, A., \& Dolan, R. J. (2004). Neural systems supporting interoceptive awareness. Nat Neurosci, 7(2), 189-195. doi:10.1038/nn1 176

Das, A., \& Menon, V. (2020). Spatiotemporal Integrity and Spontaneous Nonlinear Dynamic Properties of the Salience Network Revealed by Human Intracranial Electrophysiology: A Multicohort Replication. Cereb Cortex, 30(10), 5309-5321. doi:10.1093/cercor/bhaa111

Das, A., \& Menon, V. (2021). Asymmetric Frequency-Specific Feedforward and Feedback Information Flow between Hippocampus and Prefrontal Cortex during Verbal Memory Encoding and Recall. J Neurosci, 41(40), 8427-8440. doi:10.1523/jneurosci.080221.2021

Davis, Z. W., Muller, L., Martinez-Trujillo, J., Sejnowski, T., \& Reynolds, J. H. (2020). Spontaneous travelling cortical waves gate perception in behaving primates. Nature, 587(7834), 432-436. doi:10.1038/s41586-020-2802-y

Deen, B., Pitskel, N. B., \& Pelphrey, K. A. (2011). Three Systems of Insular Functional Connectivity Identified with Cluster Analysis. Cerebral Cortex, 21(7), 1498-1506. doi:10.1093/cercor/bhq186

Delorme, A., \& Makeig, S. (2004). EEGLAB: an open source toolbox for analysis of single-trial EEG dynamics including independent component analysis. J Neurosci Methods, 134(1), 9-21. doi:10.1016/j.jneumeth.2003.10.009

Denker, M., Zehl, L., Kilavik, B. E., Diesmann, M., Brochier, T., Riehle, A., \& Grün, S. (2018). LFP beta amplitude is linked to mesoscopic spatio-temporal phase patterns. Sci Rep, $8(1)$, 5200. doi:10.1038/s41598-018-22990-7

Desikan, R. S., Ségonne, F., Fischl, B., Quinn, B. T., Dickerson, B. C., Blacker, D., .. . Killiany, R. J. (2006). An automated labeling system for subdividing the human cerebral cortex on MRI scans into gyral based regions of interest. NeuroImage, 31(3), 968-980. doi:10.1016/j.neuroimage.2006.01.021

Dickey, C. W., Sargsyan, A., Madsen, J. R., Eskandar, E. N., Cash, S. S., \& Halgren, E. (2021). Travelling spindles create necessary conditions for spike-timing-dependent plasticity in humans. Nat Commun, 12(1), 1027. doi:10.1038/s41467-021-21298-x

Dosenbach, N. U., Fair, D. A., Cohen, A. L., Schlaggar, B. L., \& Petersen, S. E. (2008). A dualnetworks architecture of top-down control. Trends Cogn Sci, 12(3), 99-105. doi:10.1016/j.tics.2008.01.001

Dosenbach, N. U., Visscher, K. M., Palmer, E. D., Miezin, F. M., Wenger, K. K., Kang, H. C., . . . Petersen, S. E. (2006). A core system for the implementation of task sets. Neuron, 50(5), 799-812. doi:10.1016/j.neuron.2006.04.031

Engel, A. K., \& Fries, P. (2010). Beta-band oscillations--signalling the status quo? Curr Opin Neurobiol, 20(2), 156-165. doi:10.1016/j.conb.2010.02.015 
Ermentrout, G. B., \& Kleinfeld, D. (2001). Traveling electrical waves in cortex: insights from phase dynamics and speculation on a computational role. Neuron, 29(1), 33-44. doi:10.1016/s0896-6273(01)00178-7

Evrard, H. C., Forro, T., \& Logothetis, N. K. (2012). Von Economo neurons in the anterior insula of the macaque monkey. Neuron, 74(3), 482-489. doi:10.1016/j.neuron.2012.03.003

Faillenot, I., Heckemann, R. A., Frot, M., \& Hammers, A. (2017). Macroanatomy and 3D probabilistic atlas of the human insula. Neuroimage, 150, 88-98. doi:10.1016/j.neuroimage.2017.01.073

Fischl, B. (2012). FreeSurfer. Neuroimage, 62(2), 774-781. doi:10.1016/j.neuroimage.2012.01.021

Fisher, N. I. (1993). Statistical Analysis of Circular Data: Cambridge University Press.

Fudge, J. L., Breitbart, M. A., Danish, M., \& Pannoni, V. (2005). Insular and gustatory inputs to the caudal ventral striatum in primates. J Comp Neurol, 490(2), 101-118. doi:10.1002/cne.20660

Gabriel, A., \& Eckhorn, R. (2003). A multi-channel correlation method detects traveling gammawaves in monkey visual cortex. J Neurosci Methods, 131(1-2), 171-184. doi:10.1016/j.jneumeth.2003.08.008

Ghaziri, J., Tucholka, A., Girard, G., Houde, J. C., Boucher, O., Gilbert, G., . . Nguyen, D. K. (2017). The Corticocortical Structural Connectivity of the Human Insula. Cereb Cortex, 27(2), 1216-1228. doi:10.1093/cercor/bhv308

Goyal, A., Miller, J., Qasim, S. E., Watrous, A. J., Zhang, H., Stein, J. M., . . Jacobs, J. (2020). Functionally distinct high and low theta oscillations in the human hippocampus. Nat Commun, 11(1), 2469. doi:10.1038/s41467-020-15670-6

Halgren, M., Ulbert, I., Bastuji, H., Fabó, D., Erőss, L., Rey, M., . . . Cash, S. S. (2019). The generation and propagation of the human alpha rhythm. Proc Natl Acad Sci US A, 116(47), 23772-23782. doi:10.1073/pnas.1913092116

Hangya, B., Tihanyi, B. T., Entz, L., Fabó, D., Erőss, L., Wittner, L., . . . Ulbert, I. (2011). Complex propagation patterns characterize human cortical activity during slow-wave sleep. J Neurosci, 31(24), 8770-8779. doi:10.1523/jneurosci.1498-11.2011

Höistad, M., \& Barbas, H. (2008). Sequence of information processing for emotions through pathways linking temporal and insular cortices with the amygdala. Neuroimage, 40(3), 1016-1033. doi:10.1016/j.neuroimage.2007.12.043

Huntenburg, J. M., Bazin, P. L., \& Margulies, D. S. (2018). Large-Scale Gradients in Human Cortical Organization. Trends Cogn Sci, 22(1), 21-31. doi:10.1016/j.tics.2017.11.002

Jakab, A., Molnár, P. P., Bogner, P., Béres, M., \& Berényi, E. L. (2012). Connectivity-based parcellation reveals interhemispheric differences in the insula. Brain Topogr, 25(3), 264271. doi:10.1007/s10548-011-0205-y

Jenkinson, M., Bannister, P., Brady, M., \& Smith, S. (2002). Improved optimization for the robust and accurate linear registration and motion correction of brain images. Neuroimage, 17(2), 825-841. doi:10.1016/s1053-8119(02)91132-8

Jenkinson, M., \& Smith, S. (2001). A global optimisation method for robust affine registration of brain images. Med Image Anal, 5(2), 143-156. doi:10.1016/s1361-8415(01)00036-6

Jilka, S. R., Scott, G., Ham, T., Pickering, A., Bonnelle, V., Braga, R. M., . . Sharp, D. J. (2014). Damage to the Salience Network and Interactions with the Default Mode 
Network. Journal of Neuroscience, 34(33), 10798-10807.

doi:10.1523/JNEUROSCI.0518-14.2014

Johnson, R. A., \& Wehrly, T. (2020). Measures and Models for Angular Correlation and Angular-Linear Correlation. Journal of the Royal Statistical Society. Series B (Methodological), 39(2), 222-229.

Joshi, A., Scheinost, D., Okuda, H., Belhachemi, D., Murphy, I., Staib, L. H., \& Papademetris, X. (2011). Unified framework for development, deployment and robust testing of neuroimaging algorithms. Neuroinformatics, 9(1), 69-84. doi:10.1007/s12021-010-90928

King-Casas, B., Sharp, C., Lomax-Bream, L., Lohrenz, T., Fonagy, P., \& Montague, P. R. (2008). The rupture and repair of cooperation in borderline personality disorder. Science, 321(5890), 806-810. doi:10.1126/science.1156902

Kleen, J. K., Chung, J. E., Sellers, K. K., Zhou, J., Triplett, M., Lee, K., . . Chang, E. F. (2021). Bidirectional propagation of low frequency oscillations over the human hippocampal surface. Nat Commun, 12(1), 2764. doi:10.1038/s41467-021-22850-5

Kurth, F., Zilles, K., Fox, P. T., Laird, A. R., \& Eickhoff, S. B. (2010). A link between the systems: functional differentiation and integration within the human insula revealed by meta-analysis. Brain Struct Funct, 214(5-6), 519-534. doi:10.1007/s00429-010-0255-z

Lamm, C., \& Singer, T. (2010). The role of anterior insular cortex in social emotions. Brain Struct Funct, 214(5-6), 579-591. doi:10.1007/s00429-010-0251-3

Liang, Y., Song, C., Liu, M., Gong, P., Zhou, C., \& Knöpfel, T. (2021). Cortex-Wide Dynamics of Intrinsic Electrical Activities: Propagating Waves and Their Interactions. J Neurosci, 41(16), 3665-3678. doi:10.1523/jneurosci.0623-20.2021

Lozano-Soldevilla, D., \& VanRullen, R. (2019). The Hidden Spatial Dimension of Alpha: 10-Hz Perceptual Echoes Propagate as Periodic Traveling Waves in the Human Brain. Cell Rep, 26(2), 374-380.e374. doi:10.1016/j.celrep.2018.12.058

Mahjoory, K., Schoffelen, J. M., Keitel, A., \& Gross, J. (2020). The frequency gradient of human resting-state brain oscillations follows cortical hierarchies. Elife, 9. doi:10.7554/eLife.53715

Mardia, K. V. (2020). Linear-Circular Correlation Coefficients and Rhythmometry. Biometrika, 63(2), 403-405

Marks, V. S., Saboo, K. V., Topçu, Ç., Lech, M., Thayib, T. P., Nejedly, P., .. . Kucewicz, M. T. (2021). Independent dynamics of low, intermediate, and high frequency spectral intracranial EEG activities during human memory formation. Neuroimage, 245, 118637. doi:10.1016/j.neuroimage.2021.118637

Massimini, M., Huber, R., Ferrarelli, F., Hill, S., \& Tononi, G. (2004). The sleep slow oscillation as a traveling wave. J Neurosci, 24(31), 6862-6870. doi:10.1523/jneurosci.1318-04.2004

Matsui, T., Murakami, T., \& Ohki, K. (2016). Transient neuronal coactivations embedded in globally propagating waves underlie resting-state functional connectivity. Proc Natl Acad Sci U S A, 113(23), 6556-6561. doi:10.1073/pnas.1521299113

Menon, V. (2011). Large-scale brain networks and psychopathology: a unifying triple network model. Trends Cogn Sci, 15(10), 483-506. doi:10.1016/j.tics.2011.08.003

Menon, V., Gallardo, G., Pinsk, M. A., Nguyen, V. D., Li, J. R., Cai, W., \& Wassermann, D. (2020). Microstructural organization of human insula is linked to its macrofunctional circuitry and predicts cognitive control. Elife, 9. doi:10.7554/eLife.53470 
Menon, V., \& Uddin, L. Q. (2010). Saliency, switching, attention and control: a network model of insula function. Brain Structure and Function, 214(5), 655-667. doi:10.1007/s00429010-0262-0

Mesulam, M. M., \& Mufson., E. J. (1985). The insula of Reil in man and monkey. Architectonics, connectivity and function. In A. Peters \& E. G. Jones (Eds.), Cerebral cortex (pp. 179-226). New York: Plenum Pre.

Moldakarimov, S., Bazhenov, M., Feldman, D. E., \& Sejnowski, T. J. (2018). Structured networks support sparse traveling waves in rodent somatosensory cortex. Proc Natl Acad Sci U S A, 115(20), 5277-5282. doi:10.1073/pnas.1710202115

Morel, A., Gallay, M. N., Baechler, A., Wyss, M., \& Gallay, D. S. (2013). The human insula: Architectonic organization and postmortem MRI registration. Neuroscience, 236, 117 135. doi:10.1016/j.neuroscience.2012.12.076

Muller, L., Piantoni, G., Koller, D., Cash, S. S., Halgren, E., \& Sejnowski, T. J. (2016). Rotating waves during human sleep spindles organize global patterns of activity that repeat precisely through the night. Elife, 5. doi:10.7554/eLife. 17267

Muller, L., Reynaud, A., Chavane, F., \& Destexhe, A. (2014). The stimulus-evoked population response in visual cortex of awake monkey is a propagating wave. Nat Commun, 5, 3675. doi:10.1038/ncomms4675

Naidich, T. P., Kang, E., Fatterpekar, G. M., Delman, B. N., Gultekin, S. H., Wolfe, D., .. . Yousry, T. A. (2004). The insula: anatomic study and MR imaging display at $1.5 \mathrm{~T}$. AJNR Am J Neuroradiol, 25(2), 222-232.

Nauhaus, I., Busse, L., Carandini, M., \& Ringach, D. L. (2009). Stimulus contrast modulates functional connectivity in visual cortex. Nat Neurosci, 12(1), 70-76. doi:10.1038/nn.2232

Ouyang, G., Li, X., Dang, C., \& Richards, D. A. (2008). Using recurrence plot for determinism analysis of EEG recordings in genetic absence epilepsy rats. Clinical Neurophysiology, 119(8), 1747-1755. doi:10.1016/j.clinph.2008.04.005

Paquola, C., Vos De Wael, R., Wagstyl, K., Bethlehem, R. A. I., Hong, S. J., Seidlitz, J., . . . Bernhardt, B. C. (2019). Microstructural and functional gradients are increasingly dissociated in transmodal cortices. PLoS Biol, 17(5), e3000284. doi:10.1371/journal.pbio.3000284

Patel, J., Fujisawa, S., Berényi, A., Royer, S., \& Buzsáki, G. (2012). Traveling theta waves along the entire septotemporal axis of the hippocampus. Neuron, 75(3), 410-417. doi:10.1016/j.neuron.2012.07.015

Patel, J., Schomburg, E. W., Berényi, A., Fujisawa, S., \& Buzsáki, G. (2013). Local generation and propagation of ripples along the septotemporal axis of the hippocampus. J Neurosci, 33(43), 17029-17041. doi:10.1523/jneurosci.2036-13.2013

Power, J. D., Cohen, A. L., Nelson, S. M., Wig, G. S., Barnes, K. A., Church, J. A., . . Petersen, S. E. (2011). Functional network organization of the human brain. Neuron, 72(4), 665678. doi:10.1016/j.neuron.2011.09.006

Rabiner, L. R., \& Gold, B. (1975). Theory and Application of Digital Signal Processing. Englewood Cliffs, NJ: Prentice-Hall.

Raut, R. V., Snyder, A. Z., Mitra, A., Yellin, D., Fujii, N., Malach, R., \& Raichle, M. E. (2021). Global waves synchronize the brain's functional systems with fluctuating arousal. Sci $A d v, 7(30)$. doi:10.1126/sciadv.abf2709 
Rubino, D., Robbins, K. A., \& Hatsopoulos, N. G. (2006). Propagating waves mediate information transfer in the motor cortex. Nat Neurosci, 9(12), 1549-1557. doi:10.1038/nn1802

Rule, M. E., Vargas-Irwin, C., Donoghue, J. P., \& Truccolo, W. (2018). Phase reorganization leads to transient $\beta$-LFP spatial wave patterns in motor cortex during steady-state movement preparation. J Neurophysiol, 119(6), 2212-2228. doi:10.1152/jn.00525.2017

Seeley, W. W., Merkle, F. T., Gaus, S. E., Craig, A. D., Allman, J. M., \& Hof, P. R. (2012). Distinctive neurons of the anterior cingulate and frontoinsular cortex: a historical perspective. Cereb Cortex, 22(2), 245-250. doi:10.1093/cercor/bhr005

Sha, Z., Wager, T. D., Mechelli, A., \& He, Y. (2019). Common Dysfunction of Large-Scale Neurocognitive Networks Across Psychiatric Disorders. Biol Psychiatry, 85(5), 379-388. doi:10.1016/j.biopsych.2018.11.011

Singer, T., Critchley, H. D., \& Preuschoff, K. (2009). A common role of insula in feelings, empathy and uncertainty. Trends Cogn Sci, 13(8), 334-340. doi:10.1016/j.tics.2009.05.001

Spitzer, B., \& Haegens, S. (2017). Beyond the Status Quo: A Role for Beta Oscillations in Endogenous Content (Re)Activation. eNeuro, 4(4). doi:10.1523/eneuro.0170-17.2017

Sridharan, D., Levitin, D. J., \& Menon, V. (2008). A critical role for the right fronto-insular cortex in switching between central-executive and default-mode networks. Proceedings of the National Academy of Sciences, 105(34), 12569-12574. doi:10.1073/pnas.0800005105

Stefanacci, L., \& Amaral, D. G. (2002). Some observations on cortical inputs to the macaque monkey amygdala: an anterograde tracing study. J Comp Neurol, 451(4), 301-323. doi:10.1002/cne.10339

Stolk, A., Brinkman, L., Vansteensel, M. J., Aarnoutse, E., Leijten, F. S., Dijkerman, C. H., . . . Toni, I. (2019). Electrocorticographic dissociation of alpha and beta rhythmic activity in the human sensorimotor system. Elife, 8. doi:10.7554/eLife.48065

Stroh, A., Adelsberger, H., Groh, A., Rühlmann, C., Fischer, S., Schierloh, A., . . Konnerth, A. (2013). Making waves: initiation and propagation of corticothalamic $\mathrm{Ca} 2+$ waves in vivo. Neuron, 77(6), 1136-1150. doi:10.1016/j.neuron.2013.01.031

Supekar, K., \& Menon, V. (2012). Developmental maturation of dynamic causal control signals in higher-order cognition: a neurocognitive network model. PLoS Comput Biol, 8(2), e1002374. doi:10.1371/journal.pcbi.1002374

Takahashi, K., Kim, S., Coleman, T. P., Brown, K. A., Suminski, A. J., Best, M. D., \& Hatsopoulos, N. G. (2015). Large-scale spatiotemporal spike patterning consistent with wave propagation in motor cortex. Nat Commun, 6, 7169. doi:10.1038/ncomms8169

Tarjan, R. (1972). Depth-first search and linear graph algorithms. Siam J. Comput., 1(2), 146160

Townsend, R. G., Solomon, S. S., Chen, S. C., Pietersen, A. N., Martin, P. R., Solomon, S. G., \& Gong, P. (2015). Emergence of complex wave patterns in primate cerebral cortex. $J$ Neurosci, 35(11), 4657-4662. doi:10.1523/jneurosci.4509-14.2015

Uddin, L. Q. (2015). Salience processing and insular cortical function and dysfunction. Nat Rev Neurosci, 16(1), 55-61. doi:10.1038/nrn3857

Van Essen, D. C., Glasser, M. F., Dierker, D. L., Harwell, J., \& Coalson, T. (2012). Parcellations and hemispheric asymmetries of human cerebral cortex analyzed on surface-based atlases. Cereb Cortex, 22(10), 2241-2262. doi:10.1093/cercor/bhr291 
Vázquez-Rodríguez, B., Suárez, L. E., Markello, R. D., Shafiei, G., Paquola, C., Hagmann, P., . . . Misic, B. (2019). Gradients of structure-function tethering across neocortex. Proc Natl Acad Sci U S A, 116(42), 21219-21227. doi:10.1073/pnas.1903403116

Vinck, M., Lima, B., Womelsdorf, T., Oostenveld, R., Singer, W., Neuenschwander, S., \& Fries, P. (2010). Gamma-phase shifting in awake monkey visual cortex. J Neurosci, 30(4), 1250-1257. doi:10.1523/jneurosci.1623-09.2010

von Economo, C. (1926). A new type of special cells of the cingulate and insular lobes. $Z$ Ges Neurol Psychiatr, 100, 707-712.

Watrous, A. J., Miller, J., Qasim, S. E., Fried, I., \& Jacobs, J. (2018). Phase-tuned neuronal firing encodes human contextual representations for navigational goals. Elife, 7. doi:10.7554/eLife.32554

Watson, K. K., Jones, T. K., \& Allman, J. M. (2006). Dendritic architecture of the von Economo neurons. Neuroscience, 141(3), 1107-1112. doi:10.1016/j.neuroscience.2006.04.084

Wylie, K. P., Kronberg, E., Maharajh, K., Smucny, J., Cornier, M. A., \& Tregellas, J. R. (2015). Between-network connectivity occurs in brain regions lacking layer IV input. Neuroimage, 116, 50-58. doi:10.1016/j.neuroimage.2015.05.010

$\mathrm{Xu}, \mathrm{W}$., Huang, X., Takagaki, K., \& Wu, J. Y. (2007). Compression and reflection of visually evoked cortical waves. Neuron, 55(1), 119-129. doi:10.1016/j.neuron.2007.06.016

Yushkevich, P. A., Piven, J., Hazlett, H. C., Smith, R. G., Ho, S., Gee, J. C., \& Gerig, G. (2006). User-guided 3D active contour segmentation of anatomical structures: significantly improved efficiency and reliability. Neuroimage, 31(3), 1116-1128. doi:10.1016/j.neuroimage.2006.01.015

Zanos, T. P., Mineault, P. J., Nasiotis, K. T., Guitton, D., \& Pack, C. C. (2015). A sensorimotor role for traveling waves in primate visual cortex. Neuron, 85(3), 615-627. doi:10.1016/j.neuron.2014.12.043

Zhang, H., \& Jacobs, J. (2015). Traveling Theta Waves in the Human Hippocampus. J Neurosci, 35(36), 12477-12487. doi:10.1523/jneurosci.5102-14.2015

Zhang, H., Watrous, A. J., Patel, A., \& Jacobs, J. (2018). Theta and Alpha Oscillations Are Traveling Waves in the Human Neocortex. Neuron, 98(6), 1269-1281.e1264. doi:10.1016/j.neuron.2018.05.019

Zhang, J., Abiose, O., Katsumi, Y., Touroutoglou, A., Dickerson, B. C., \& Barrett, L. F. (2019). Intrinsic Functional Connectivity is Organized as Three Interdependent Gradients. Sci Rep, 9(1), 15976. doi:10.1038/s41598-019-51793-7 


\section{Figures}

a
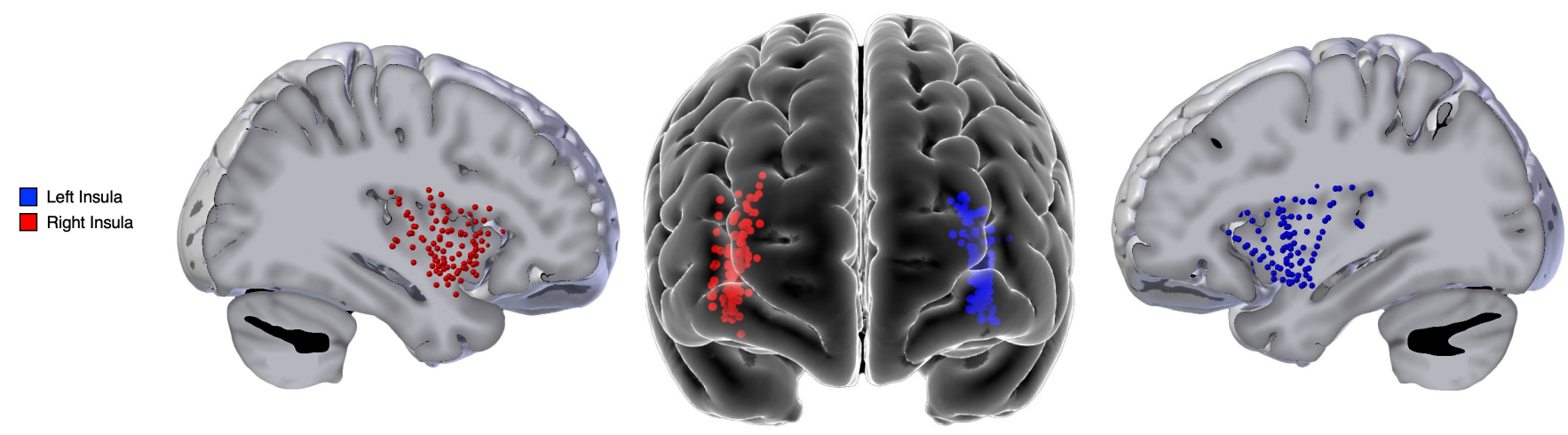

b

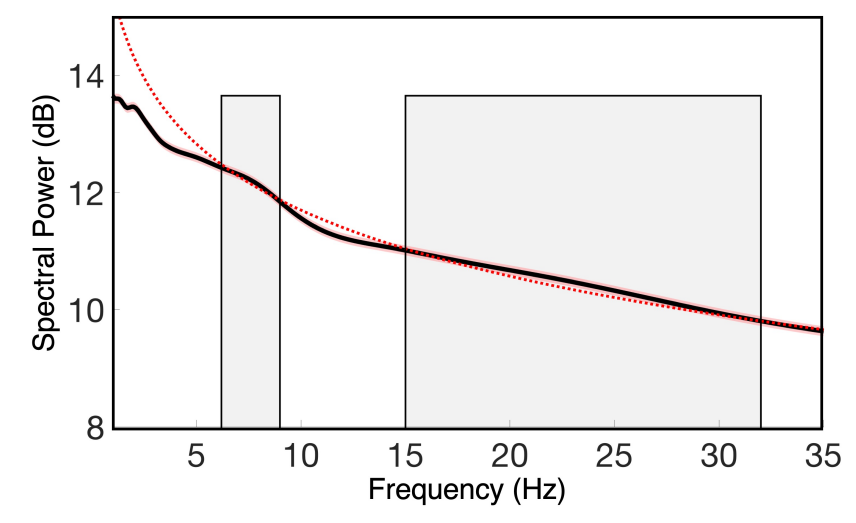

C
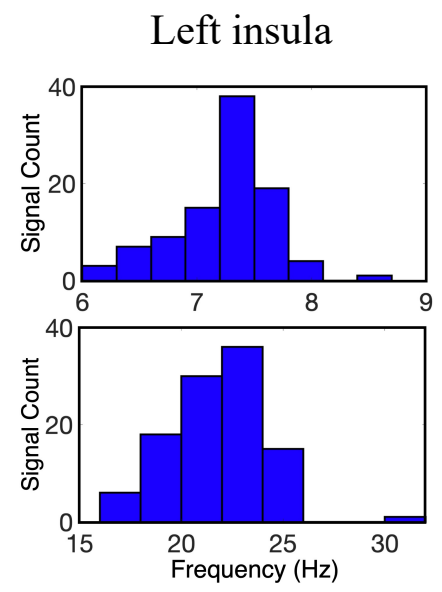

.

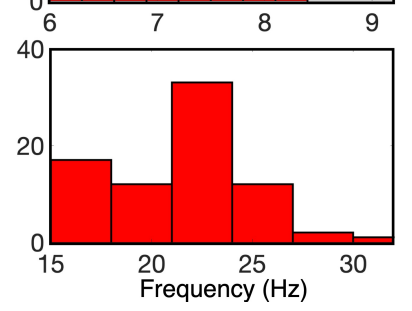

Figure 1: Theta and beta band frequency oscillations in the human insula. (a) Lateral and frontal views of the brain showing electrodes in the left $(n=125)$ and right insula $(n=114)$ across all participants. (b) An oscillation-detection algorithm known as the MODAL detected theta $(\sim 6-9 \mathrm{~Hz})$ and beta $(\sim 15-32 \mathrm{~Hz})$ oscillations across all electrodes in the insula (Methods). The black line depicts the power spectrum, the red dotted line depicts the $1 / \mathrm{f}$ regression line, and the shaded regions indicate the detected frequency bands at which the power spectrum was higher than the $1 / \mathrm{f}$ background signal. (c) The distribution of the peak frequencies detected across all insula electrodes. In the left insula, $90 \%$ of electrodes had theta oscillations and $99 \%$ had beta oscillations. In the right insula, $80 \%$ of electrodes had theta oscillations and $81 \%$ had beta oscillations. 


\section{Left insula}

$\mathrm{a}$

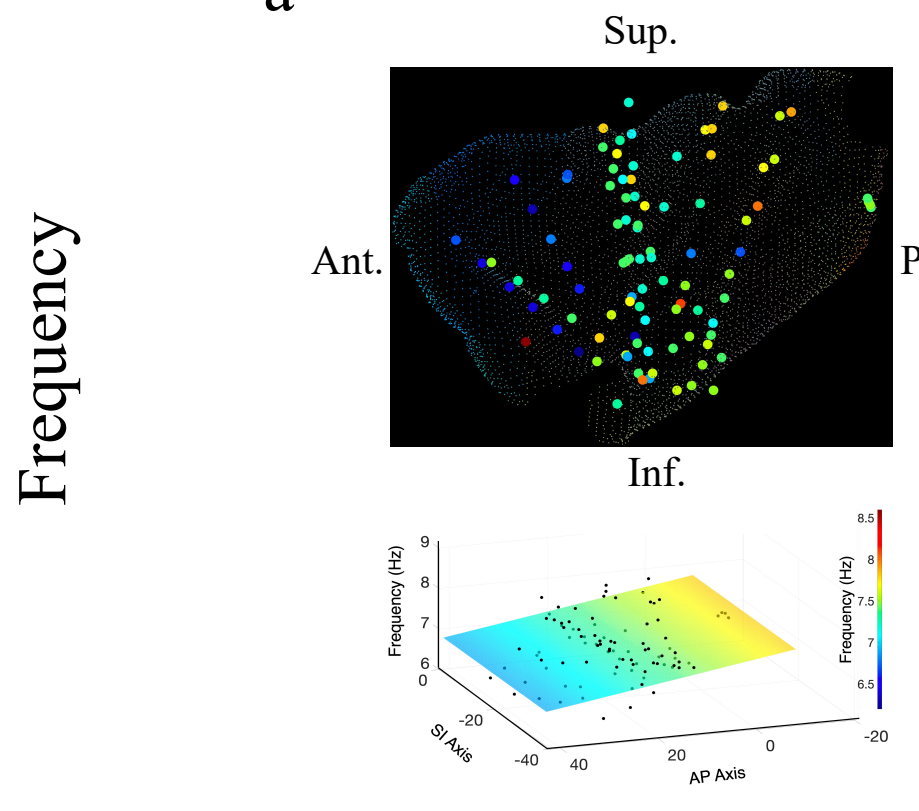

$\mathrm{C}$
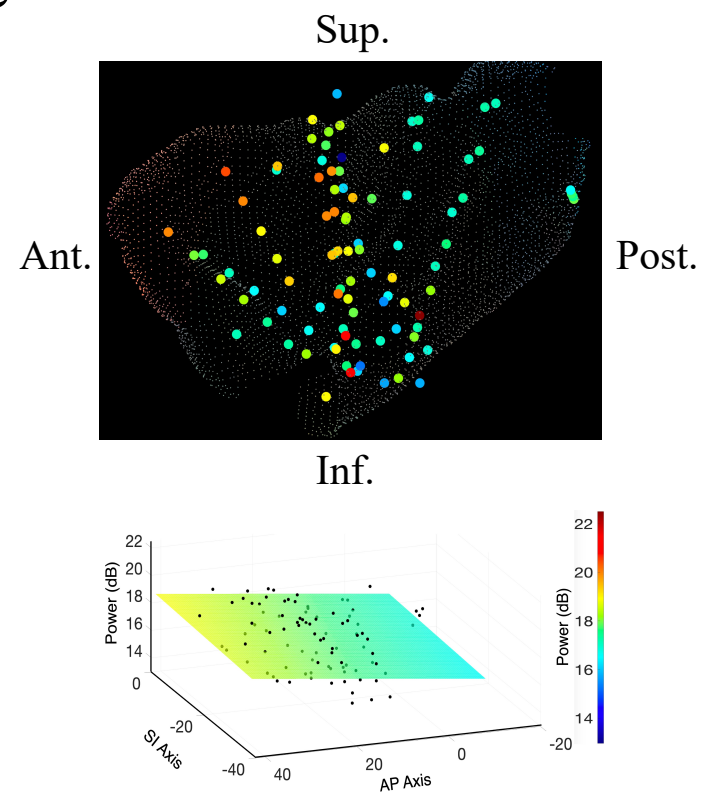

Right insula

b
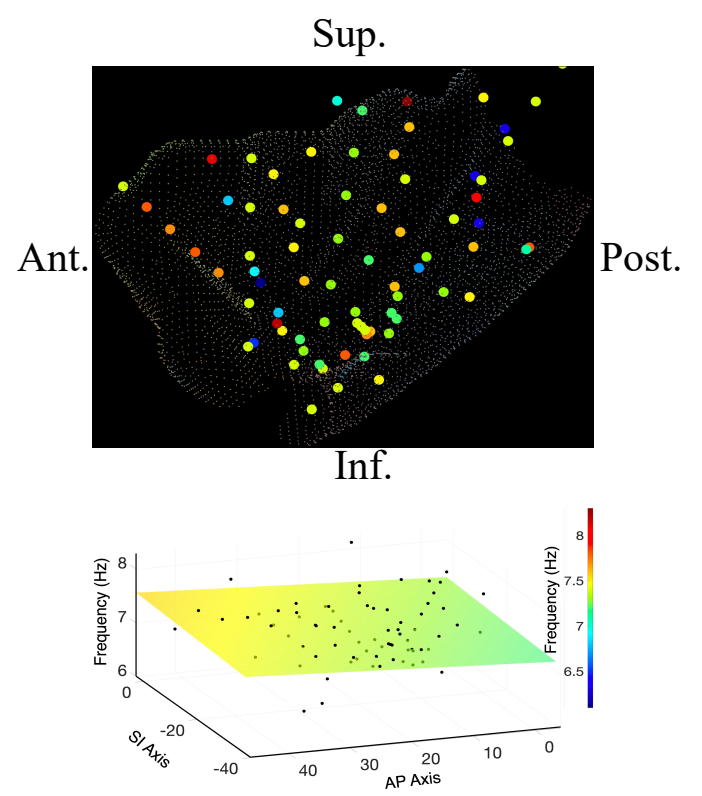

d
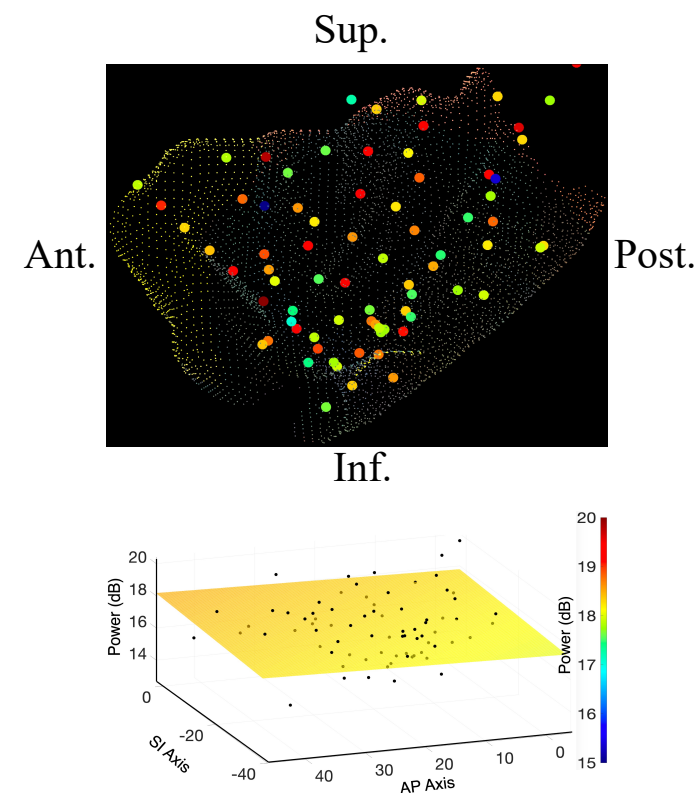

Figure 2: Frequency and power gradients of theta oscillations in the insula. (a) Peak frequencies within the theta band ( 6-9 Hz) showed posterior-to-anterior gradient in the left insula. Coordinates of electrodes along the AP axis predicted peak theta frequency, where slower theta frequencies were more prominent in the anterior insula and faster theta frequencies were prominent posteriorly $(t(74)=-4.09, p<0.001)$. (b) Theta frequencies in the right insula were not hierarchically organized along the AP axis $(t(57)=-0.14$, $p>0.05)$, or the SI axis $(t(57)=0.07, p>0.05)$. (c) Theta power showed anterior-to-posterior gradient in the left insula with theta power being higher in the anterior insula $(t(74)=2.52, p<0.05)$. (d) Theta power showed a superior-to-inferior gradient in the right insula where higher amplitude theta oscillations were prominent in the superior portion of the insula $(t(57)=2.92, p<0.01)$. A marginally significant AP gradient suggests that theta power was slightly more concentrated in the right anterior insula, similar to the power gradient in the left hemisphere $(t(57)=1.98, p=0.053)$. No interaction effect was observed between AP and SI axes $(t(57)=-$ $1.14, p>0.05$ ). Results were computed using linear regression where the y-axis (AP) and z-axis (SI) electrode coordinates were used as continuous independent variables to predict spectral power and frequency dynamics. Variability across participants has been accounted for in all t-statistics. Images were generated via automatic selection of insular vertices from anatomical parcellations using the Desikan-Killiany (DK) atlas. 


\section{Left insula}

a
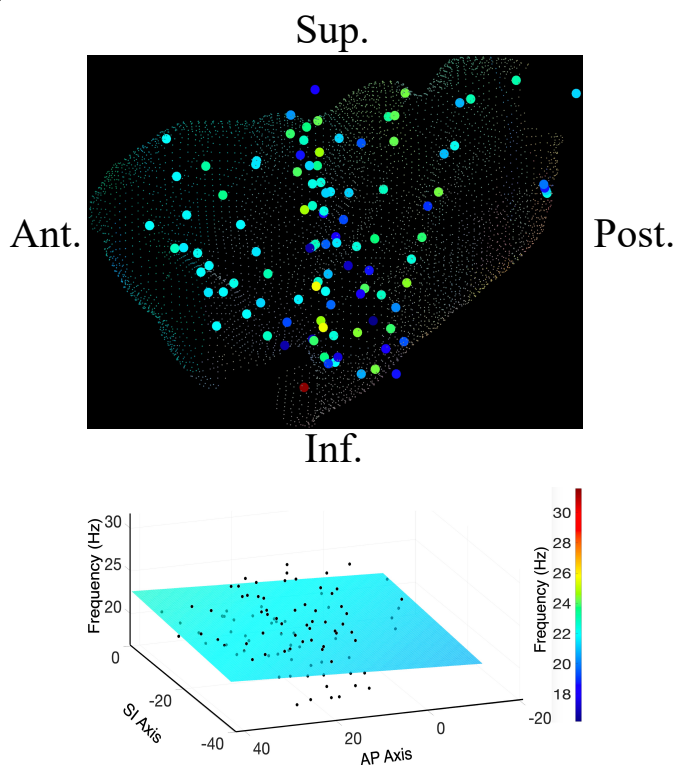

$\mathrm{C}$

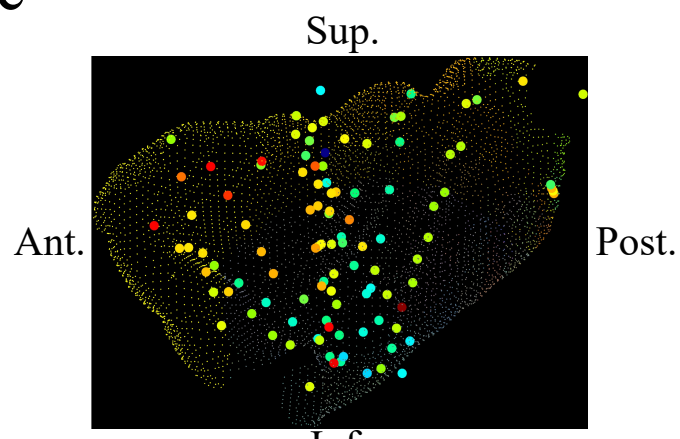

Inf.

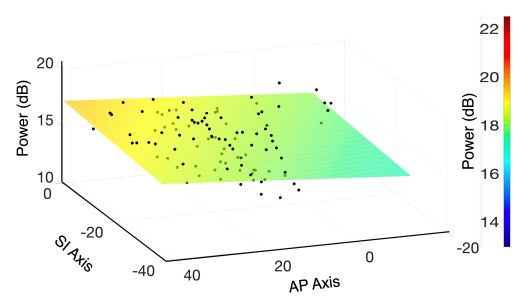

Right insula

$\mathrm{b}$
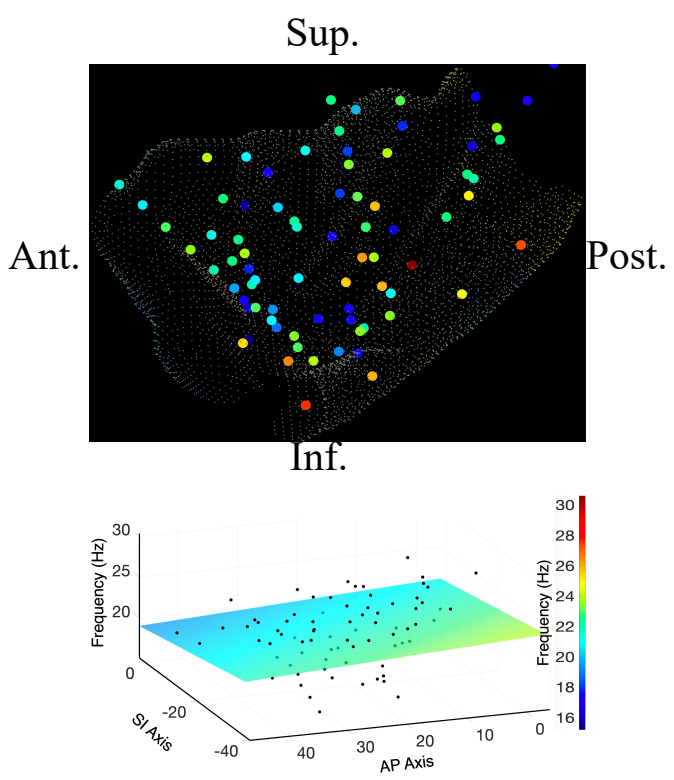

$\mathrm{d}$
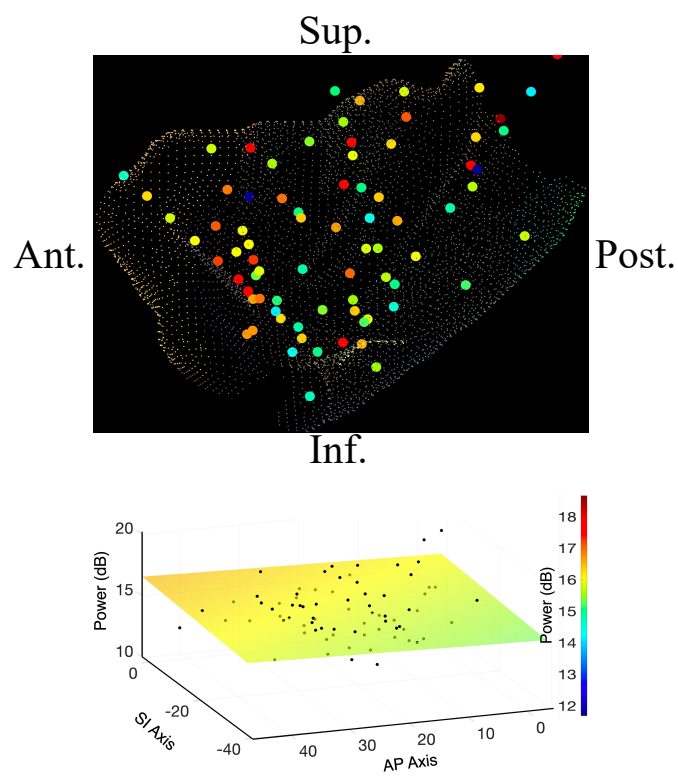

Figure 3: Frequency and power gradients of beta oscillations in the insula. (a) Peak frequencies within the beta band ( 15-32 Hz) showed no spatial organization in the left insula $(t(84)=-0.95, p>0.05)$. (b) Beta frequencies in the right insula did not show AP $(t(58)=-0.63, p>0.05)$, or SI gradients $(t(58)=-1.10, p>0.05)$. (c) Electrode coordinates along the AP axis of the left insula predicted beta power, revealing that higher power oscillations were prominent in the anterior insula $(t(84)=3.07, p<0.01)$. (d) Beta power in the right insula also showed an AP gradient where higher amplitude beta oscillations were prominent in the right anterior portion $(t(58)=2.97, p<0.01)$. Results were computed using linear regression where the y-axis (AP) and z-axis (SI) electrode coordinates were used as continuous independent variables to predict spectral power and frequency dynamics. Variability across participants has been accounted for in all t-statistics. Images were generated via automatic selection of insular vertices from anatomical parcellations using the DK atlas. 
a
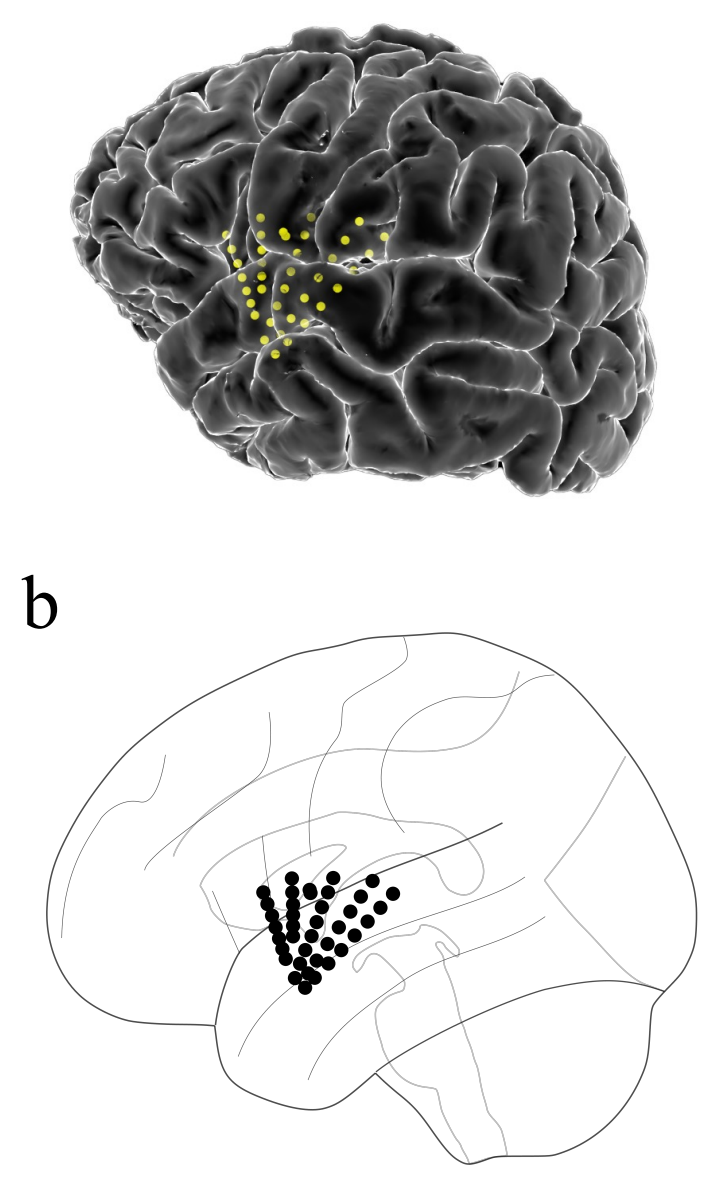

\section{c}
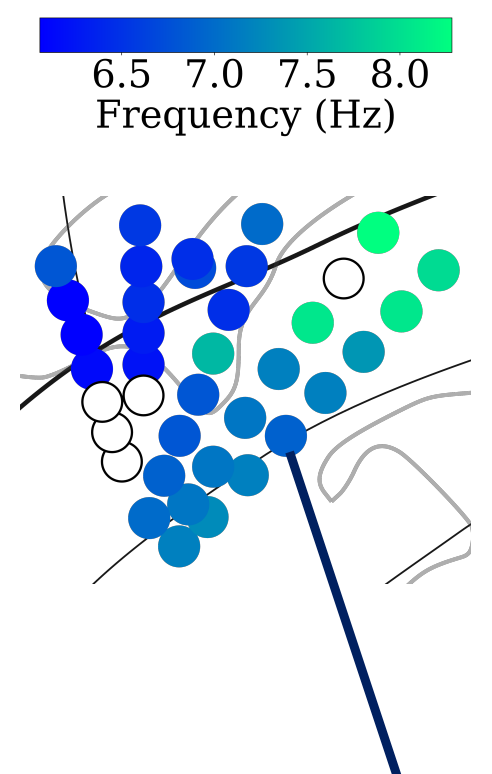

d

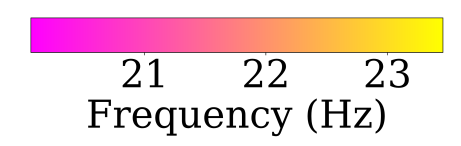

f

e

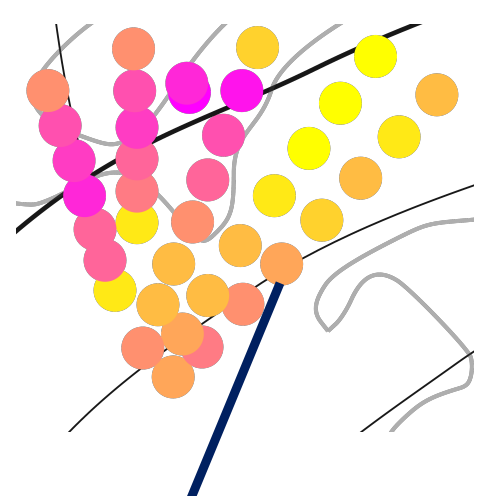

f

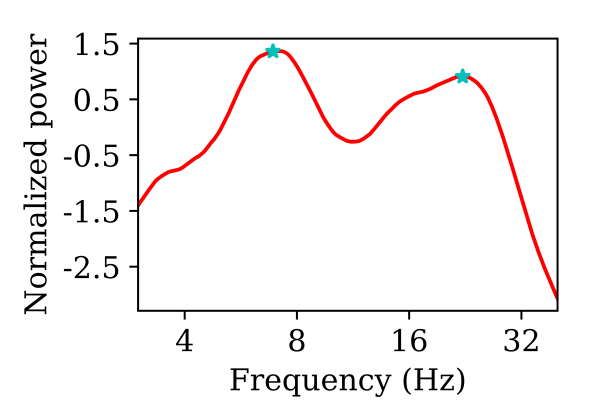

Figure 4: Example of spatiotemporal clustering in the human insula. A cluster was identified as a spatially contiguous groups of electrodes that had narrowband oscillations at either theta or beta frequency band (Methods). (a) Insula electrodes visualized in a brain surface plot in participant \#3. (b) Insula electrodes projected on to a flat brain which was subsequently used for visualization of oscillation clusters and traveling waves. (c) Theta frequency oscillation cluster in participant \#3 (electrodes with black circles denote electrodes without any narrowband peaks in the power spectrum). (d) Beta frequency oscillation cluster in the same participant. (e) Mean power spectrum (blue) in log-log coordinates from an example electrode in participant \#3. Black line denotes fitted $1 / \mathrm{f}$ line to the mean power spectrum in $\log -\log$ coordinates using robust linear regression (see Methods). (f) Normalized power spectrum (red) after removal of the 1/f background signal. This approach emphasizes narrowband oscillations as positive deflections. Stars (cyan) denote the narrowband peaks in the normalized power spectrum, identified as any local maximum greater than one standard deviation above the mean (see Methods). 
bioRxiv preprint doi: https://doi.org/10.1101/2021.12.16.473034; this version posted December 19, 2021. The copyright holder for this preprint (which was not certified by peer review) is the author/funder, who has granted bioRxiv a license to display the preprint in perpetuity. It is made available under aCC-BY-NC-ND 4.0 International license.

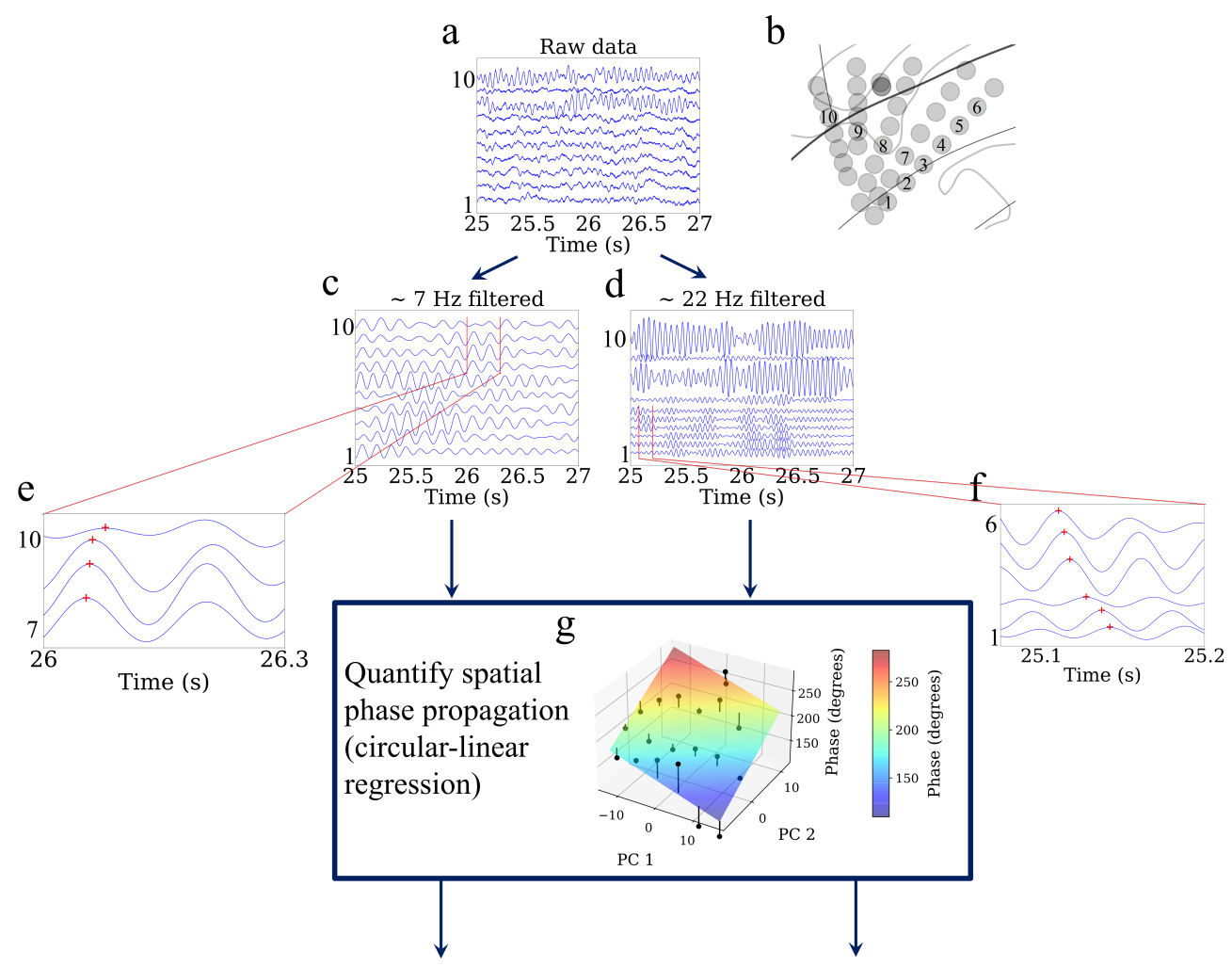

$\mathrm{h}$

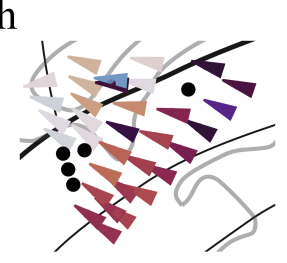

Low frequency traveling wave

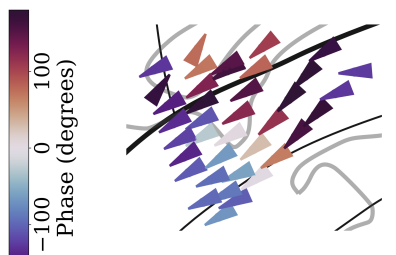

High frequency traveling wave i

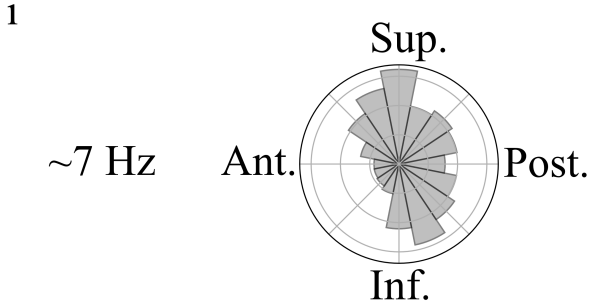

j

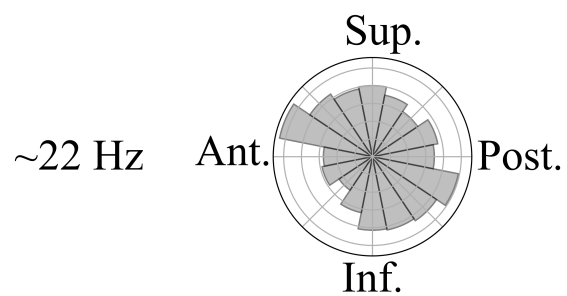

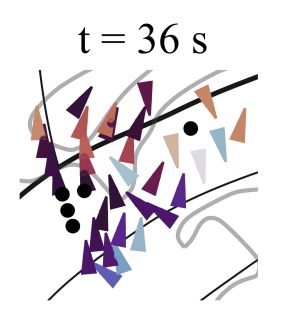
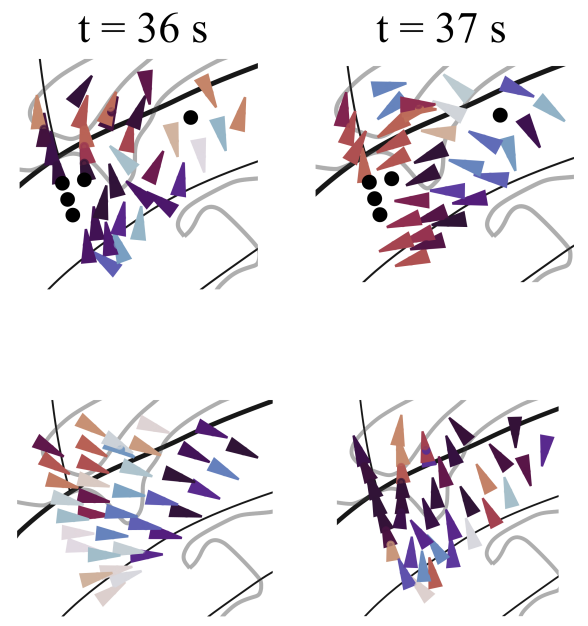

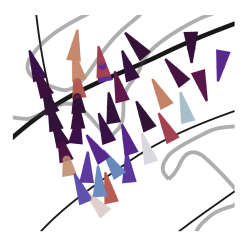

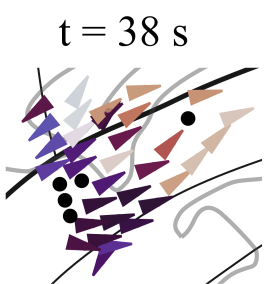

$\mathrm{t}=39 \mathrm{~s}$
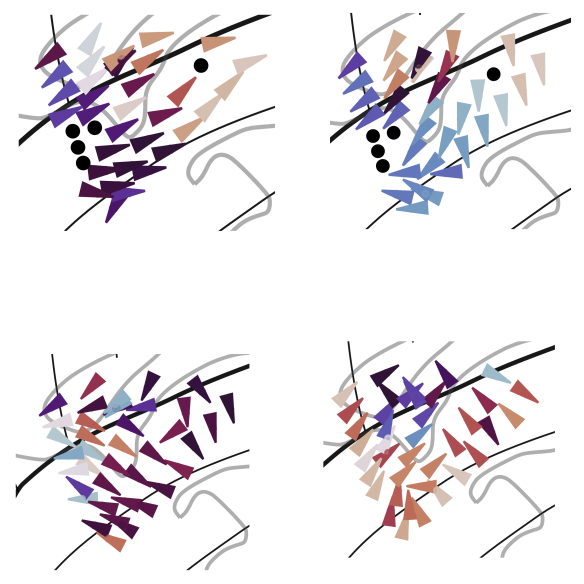

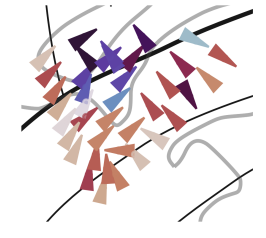

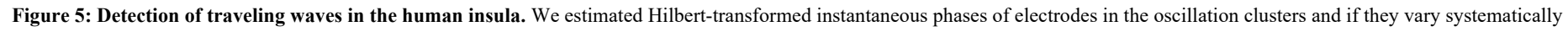

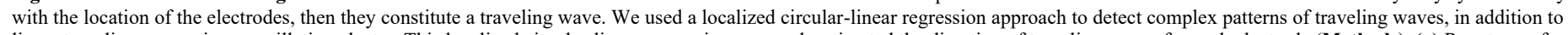

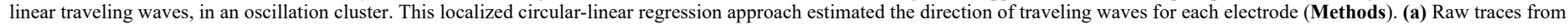

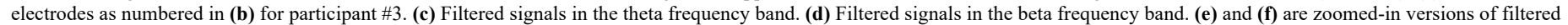

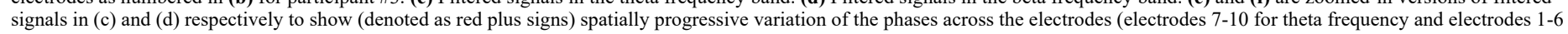

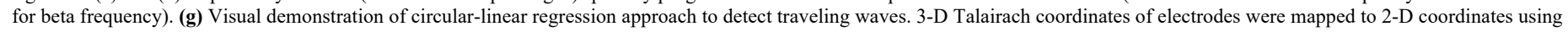

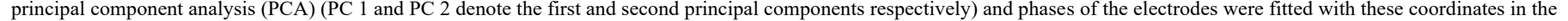

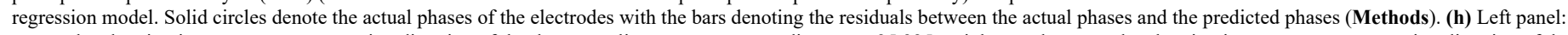

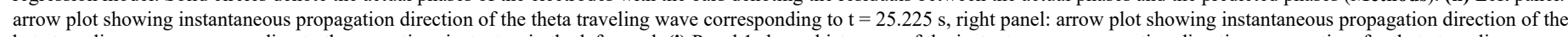

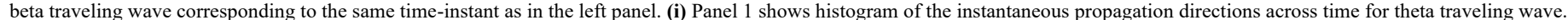

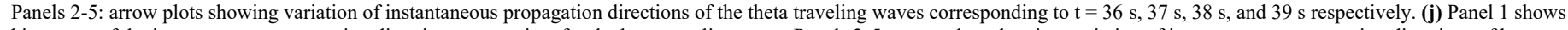
histogram of the instantaneous propagation directions across time for the beta traveling wave. Panels 2-5: arrow plots showing variation of instantaneous propagation directions of beta traveling waves corresponding to $t=36$ s, 37 s, 38 s, and 39 s respectively. Ant.: Anterior, Post.: Posterior, Sup.: Superior, Inf.: Inferior. 

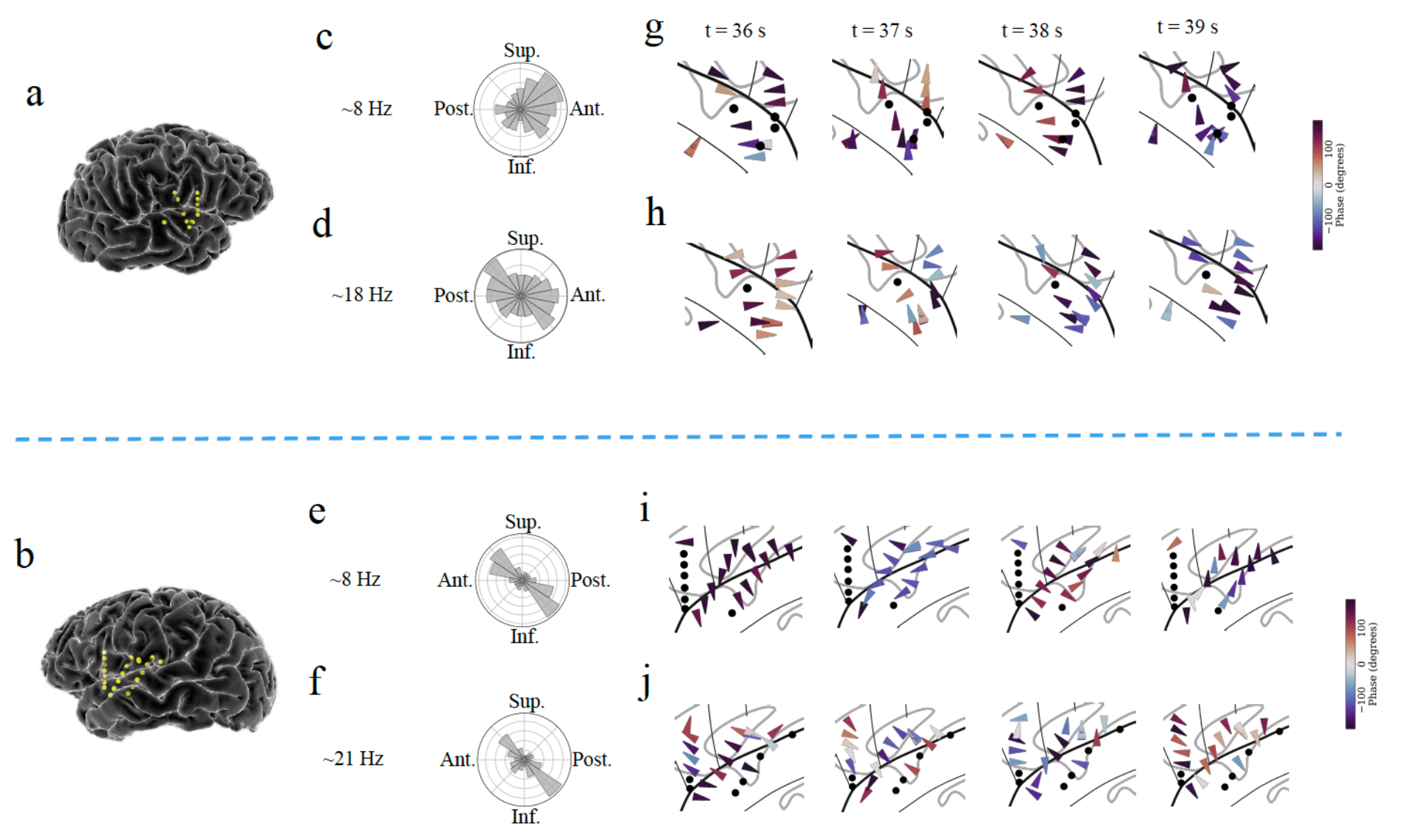

Figure 6: Example of traveling waves in the human insula. Traveling waves changed their directions with respect to time. Insula electrodes visualized in a brain surface plot in (a) participant \#6 and (b) participant \#7. (c) and (d) are histograms of the instantaneous propagation directions across time for theta $(\sim 8 \mathrm{~Hz})$ and beta $(\sim 18 \mathrm{~Hz})$ traveling waves in participant \#6. (e) and (f) are the same as in (c) and (d) respectively, for participant \#7. (g) Panels 1-4: arrow plots showing variation of instantaneous propagation direction of theta traveling waves corresponding to $t=36 \mathrm{~s}, 37 \mathrm{~s}, 38 \mathrm{~s}$, and $39 \mathrm{~s}$ respectively in participant \#6, visualized in a zoomed-in version of flat brain. (h) Panels 1-4: arrow plots showing instantaneous propagation directions of beta traveling waves corresponding to $\mathrm{t}=36 \mathrm{~s}, 37 \mathrm{~s}, 38 \mathrm{~s}$, and $39 \mathrm{~s}$ respectively in participant \#6, visualized in a zoomed-in version of flat brain. (i) and (j) are same as in (g) and (h) respectively, for participant \#7. Solid circles represent electrodes without narrowband oscillations. Ant.: Anterior, Post.: Posterior, Sup.: Superior, Inf.: Inferior. 
bioRxiv preprint doi: https://doi.org/10.1101/2021.12.16.473034; this version posted December 19, 2021. The copyright holder for this preprint (which was not certified by peer review) is the author/funder, who has granted bioRxiv a license to display the preprint in perpetuity. It is made available under aCC-BY-NC-ND 4.0 International license.

a

d

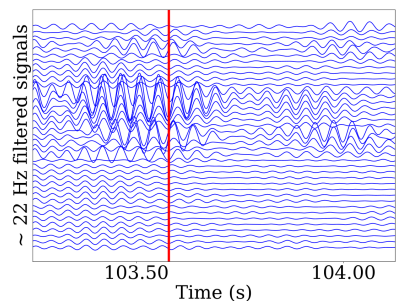

b
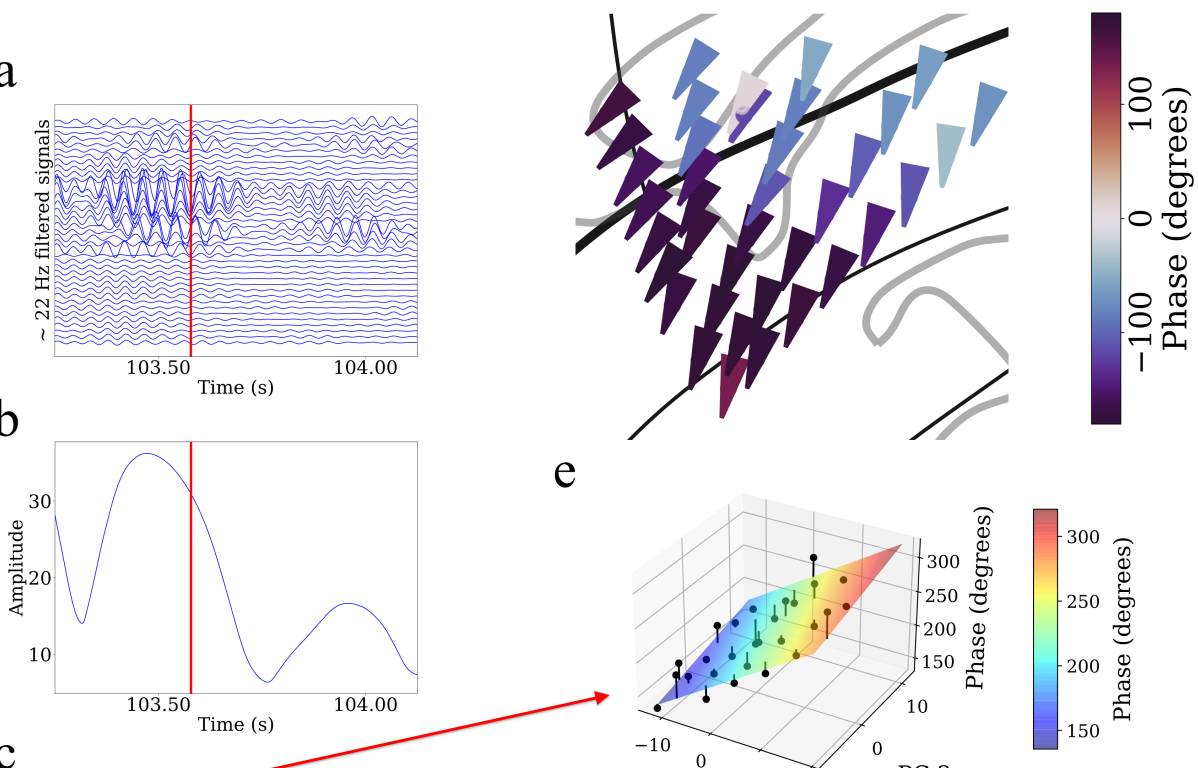

e
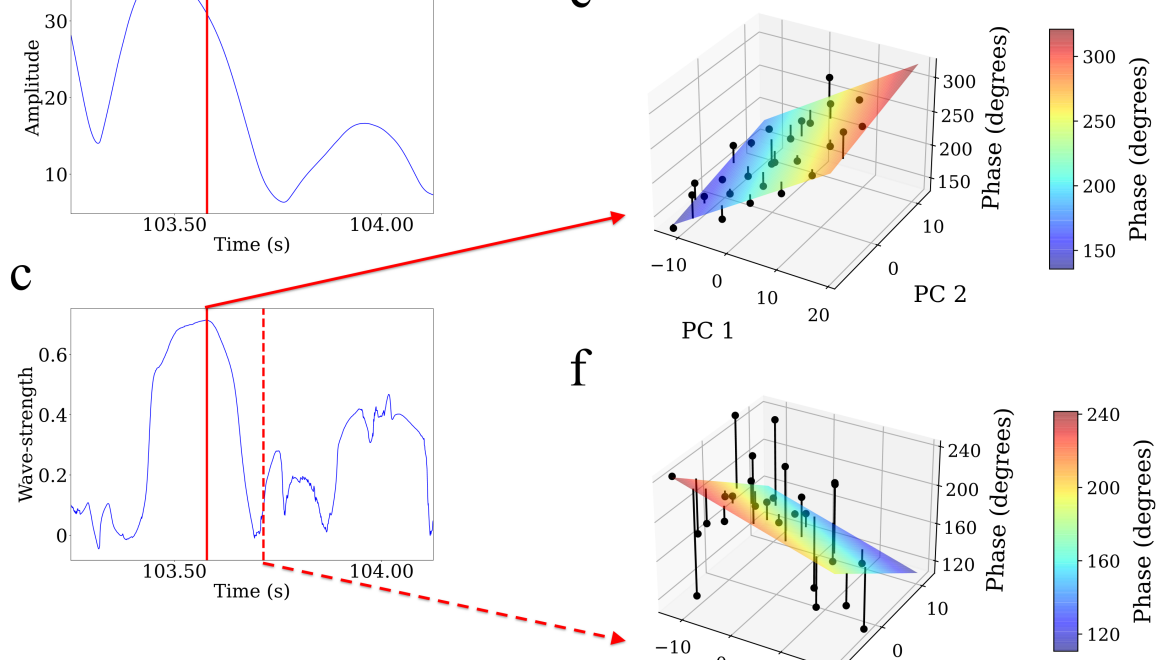

PC 1
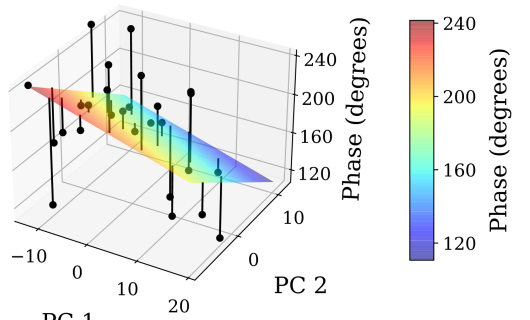

PC 1

g

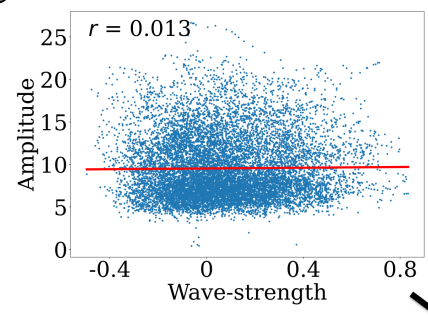

h

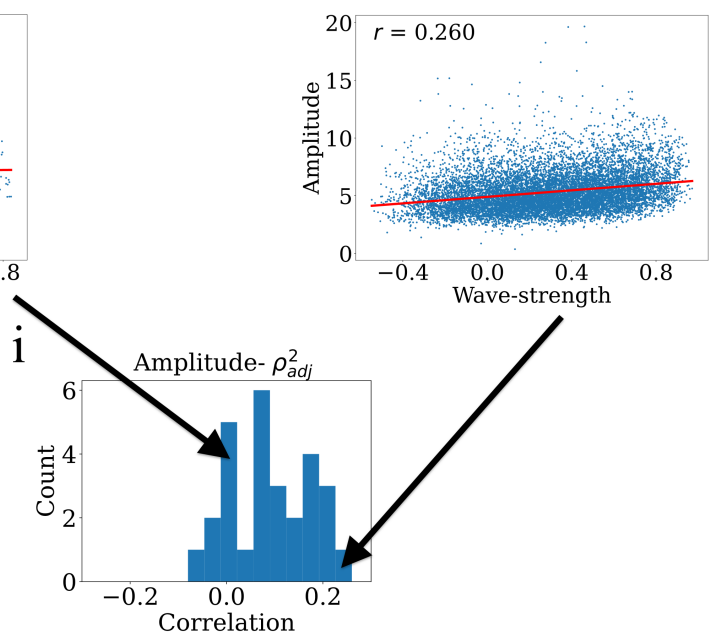

Figure 7: Example showing that higher wave-strength values of traveling waves correspond to higher Hilbert-transformed amplitude of signals in participant \#3. (a) Filtered signals in the beta frequency band from all electrodes in participant $\# 3$ in time interval $103.25 \mathrm{~s}$ to $104.125 \mathrm{~s}$. (b) Average amplitude across all electrodes in the same time interval. We defined the average amplitude for each oscillation cluster to be the average of the Hilbert-transformed instantaneous amplitudes of filtered signals of all electrodes. (c) Wave-strength, averaged across all electrodes, in the same time interval. Higher wave-strength corresponds to higher amplitudes of electrodes in (b) (But observe that the amplitude peaks earlier than the wave-strength; see Results). Time-instant corresponding to the local maximum of wave-strength in (c) is denoted by red lines in (a), (b), and (c). (d) Arrow plot showing instantaneous propagation direction of electrodes for the beta traveling wave at the time-instant corresponding to the red line in (c). Note the smooth variation of phases across space, which results in the high value of wave-strength in (c). (e) Circular-linear regression fit at the time-instant corresponding to the red line in (c). Solid circles denote the actual phases of the electrodes with the bars denoting the residuals between the actual phases and the predicted phases. Note the relatively low values of the residuals indicated by the low heights of the bars. (f) Circular-linear regression fit at a time-instant (denoted as dotted red line in (c)) for which the wave-strength is relatively low. Note the relatively high values of the residuals. (g)-(i) Strength (denoted as $\rho_{a d j}^{2}$ ) of traveling waves is positively correlated with Hilbert-transformed amplitude of signals. We estimated the average (averaged across electrodes) Hilbert-transformed instantaneous amplitudes of signals of the electrodes for each oscillation cluster and estimated their correlation with the wave-strength of traveling waves. We used a circular-shuffling procedure to test the significance of correlations where we circularly shuffle the wave-strength values with respect to the average amplitude and estimate the correlation on this shuffled data to build a distribution of surrogate correlation values against which the observed correlation was tested $(p<0.05)$ (Methods). (g) Amplitude- $\rho_{a d j}^{2}$ scatter plot for cluster 1 of participant \#7 where there was no statistically significant correlation $(p>$

0.05). (h) Amplitude- $\rho_{a d j}^{2}$ scatter plot for cluster 2 of participant \#8 where there was a statistically significant correlation $(p<0.05)$. (i) Histogram of correlations across all clusters (also see

Table S1). 
bioRxiv preprint doi: $\mathrm{https}$ //doi.org/10.1101/2021.12 16.473034; this version posted December 19,2021 . The copyright holder for this preprint (which was not certified by peer review) is the author/funder, who has granted bioRxiv a license to display the preprint in perpetuity. It is made available under aCC-BY-NC-ND 4.0 International license.

\section{Supplementary figures}

\section{Figure S1: Oscillation clusters in participant \#1.}

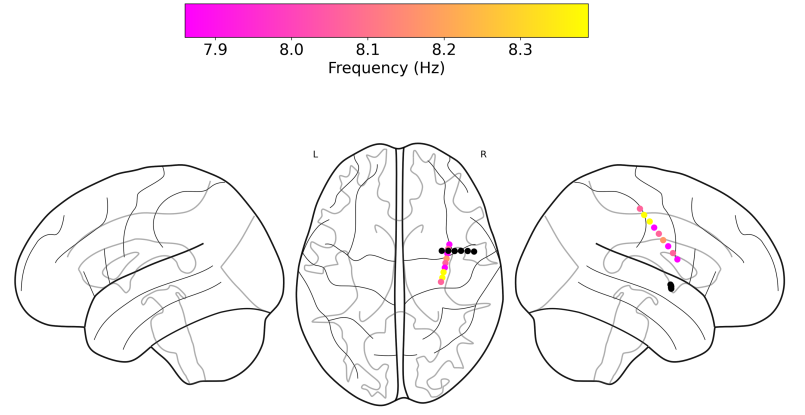

Subject \# 1, cluster \# 1, cluster frequency $=8.081 \mathrm{~Hz}, \mathrm{n}=9$
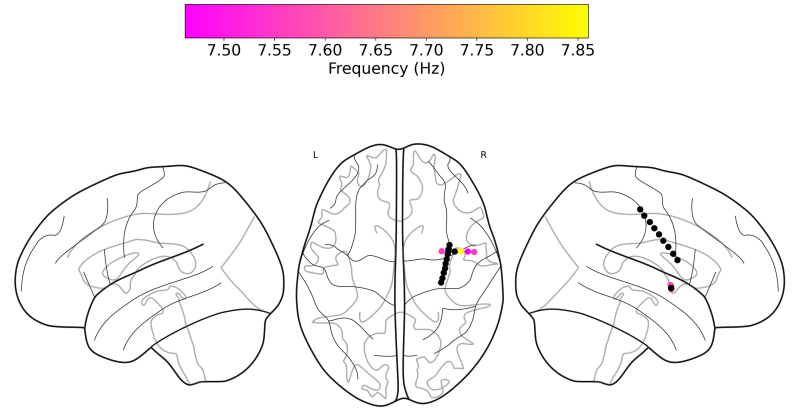

Subject \# 1, cluster \# 3, cluster frequency $=7.61 \mathrm{~Hz}, \mathrm{n}=4$

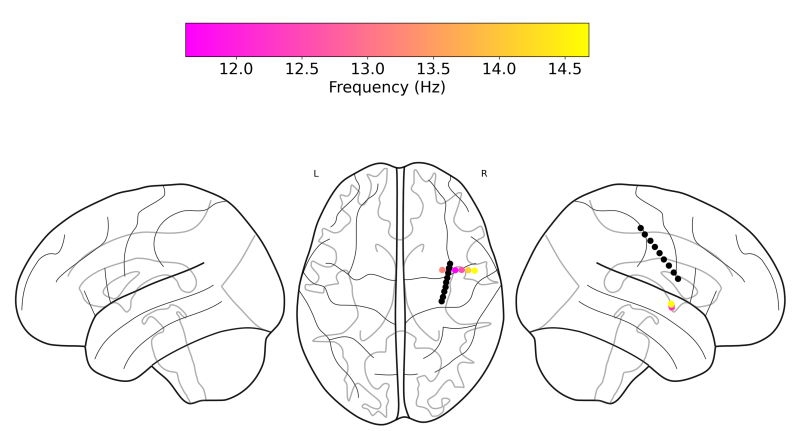

Subject \# 1, cluster \# 2, cluster frequency $=12.97 \mathrm{~Hz}, \mathrm{n}=6$
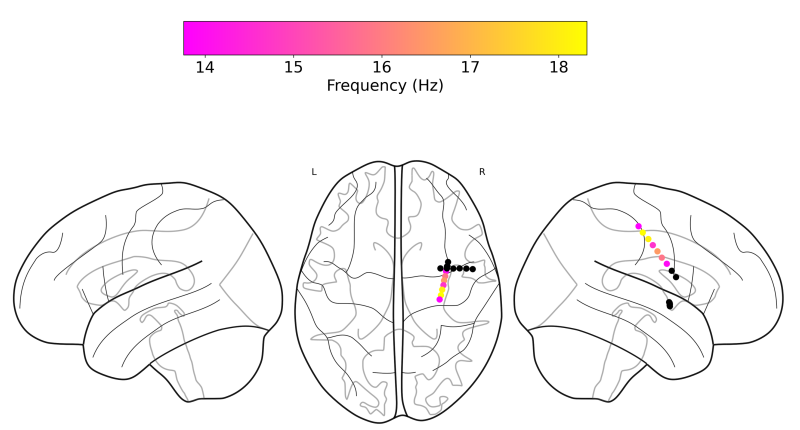

Subject \# 1, cluster \# 4, cluster frequency $=15.879 \mathrm{~Hz}, \mathrm{n}=7$ 
bioRxiv preprint doi: $\mathrm{https}$ //doi.org/10.1101/2021.12.16.473034; this version posted December 19, 2021. The copyright holder for this preprint (which was not certified by peer review) is the author/funder, who has granted bioRxiv a license to display the preprint in perpetuity. It is made available under aCC-BY-NC-ND 4.0 International license.

\section{Figure S2: Oscillation clusters in participant \#2.}

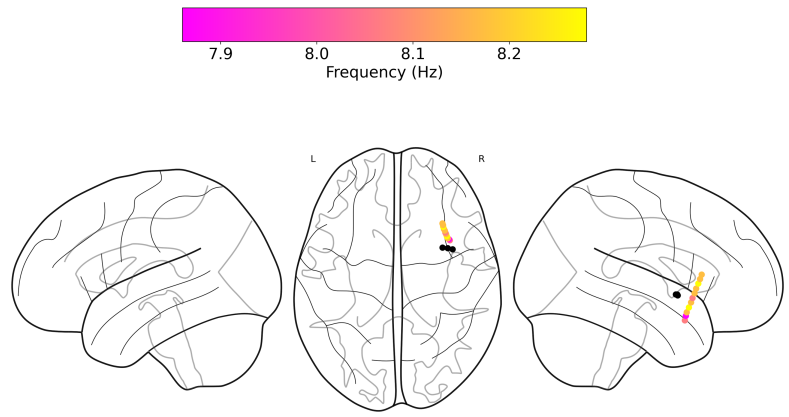

Subject \# 2, cluster \# 1, cluster frequency $=8.145 \mathrm{~Hz}, \mathrm{n}=11$

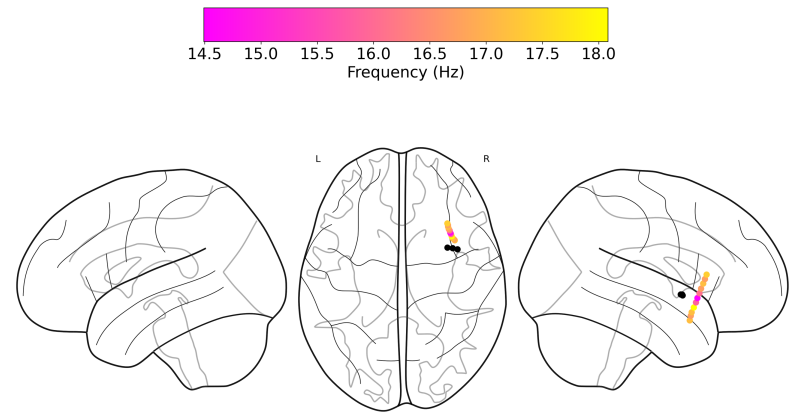

Subject \# 2, cluster \# 2, cluster frequency $=16.689 \mathrm{~Hz}, \mathrm{n}=11$

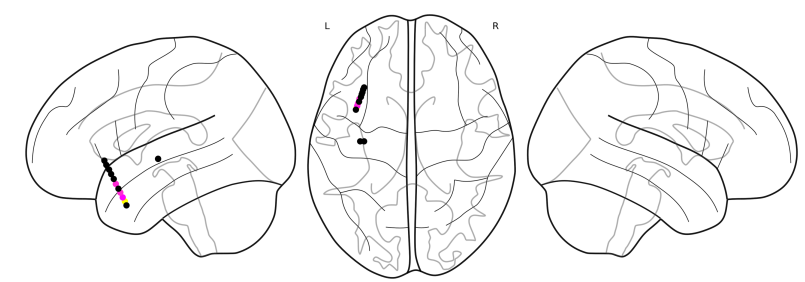

Subject \# 2, cluster \# 3, cluster frequency $=4.662 \mathrm{~Hz}, \mathrm{n}=4$ 
bioRxiv preprint doi: https://doi.org/10.1101/2021.12.16.473034; this version posted December 19, 2021. The copyright holder for this preprint (which was not certified by peer review) is the author/funder, who has granted bioRxiv a license to display the preprint in perpetuity. It is made available under aCC-BY-NC-ND 4.0 International license.

Figure S3: Oscillation clusters in participant \#3.
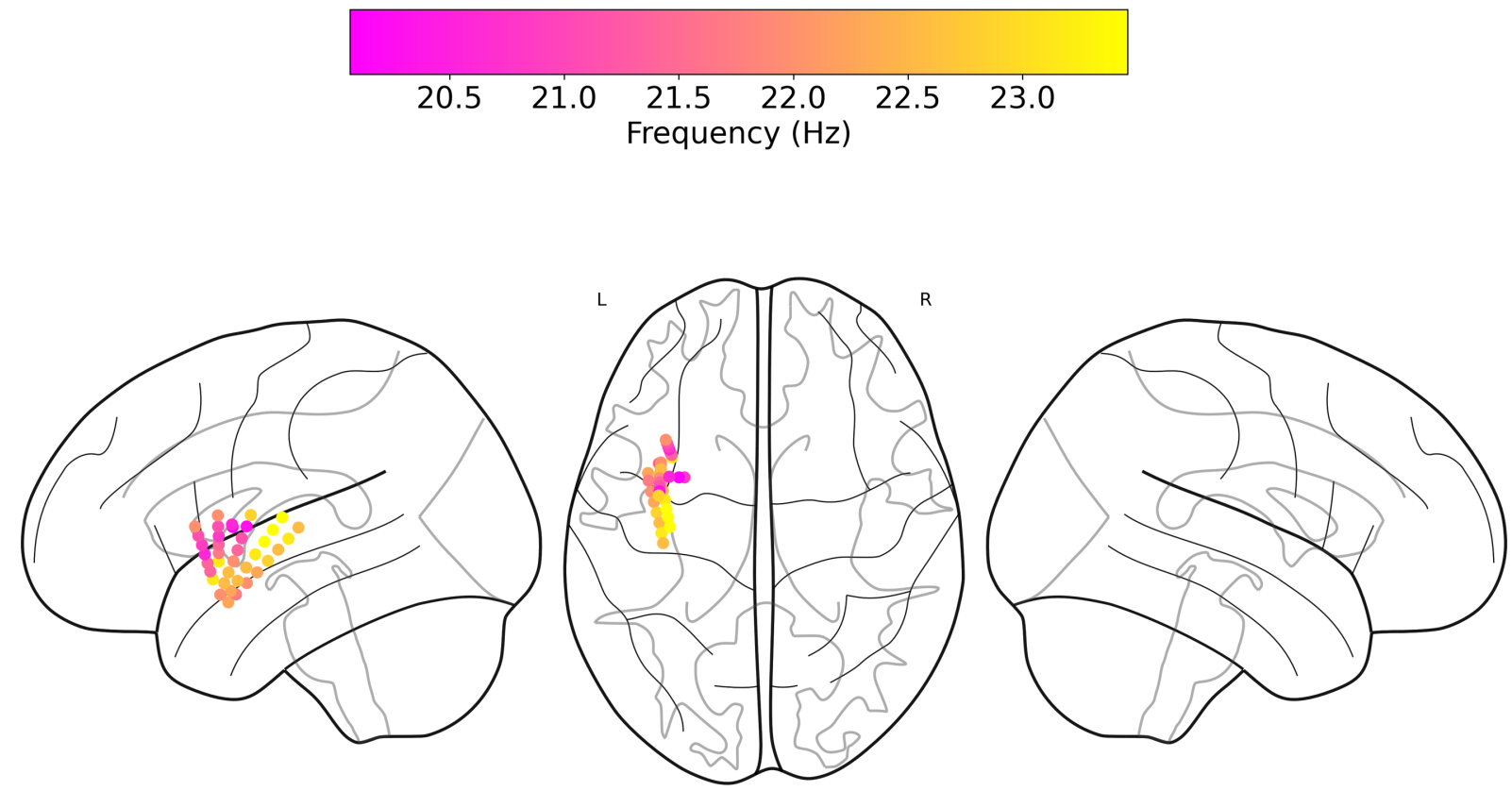

Subject \# 3, cluster \# 1, cluster frequency $=21.984 \mathrm{~Hz}, \mathrm{n}=39$
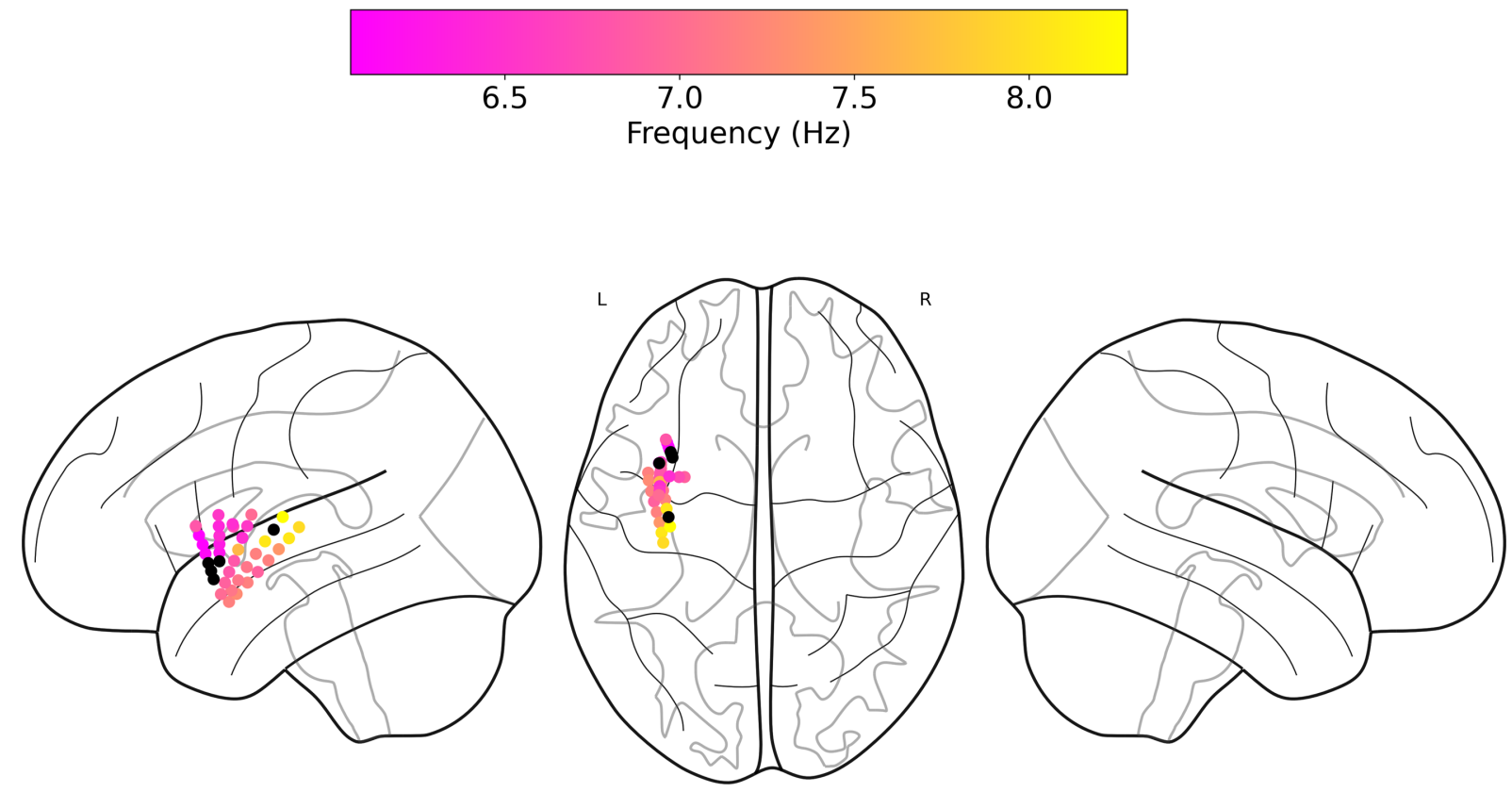

Subject \# 3, cluster \# 2, cluster frequency $=6.944 \mathrm{~Hz}, \mathrm{n}=34$ 
bioRxiv preprint doi: https://doi.org/10.1101/2021.12.16.473034; this version posted December 19, 2021. The copyright holder for this preprint (which was not certified by peer review) is the author/funder, who has granted bioRxiv a license to display the preprint in perpetuity. It is made available under aCC-BY-NC-ND 4.0 International license.

Figure S4: Oscillation clusters in participant \#5.
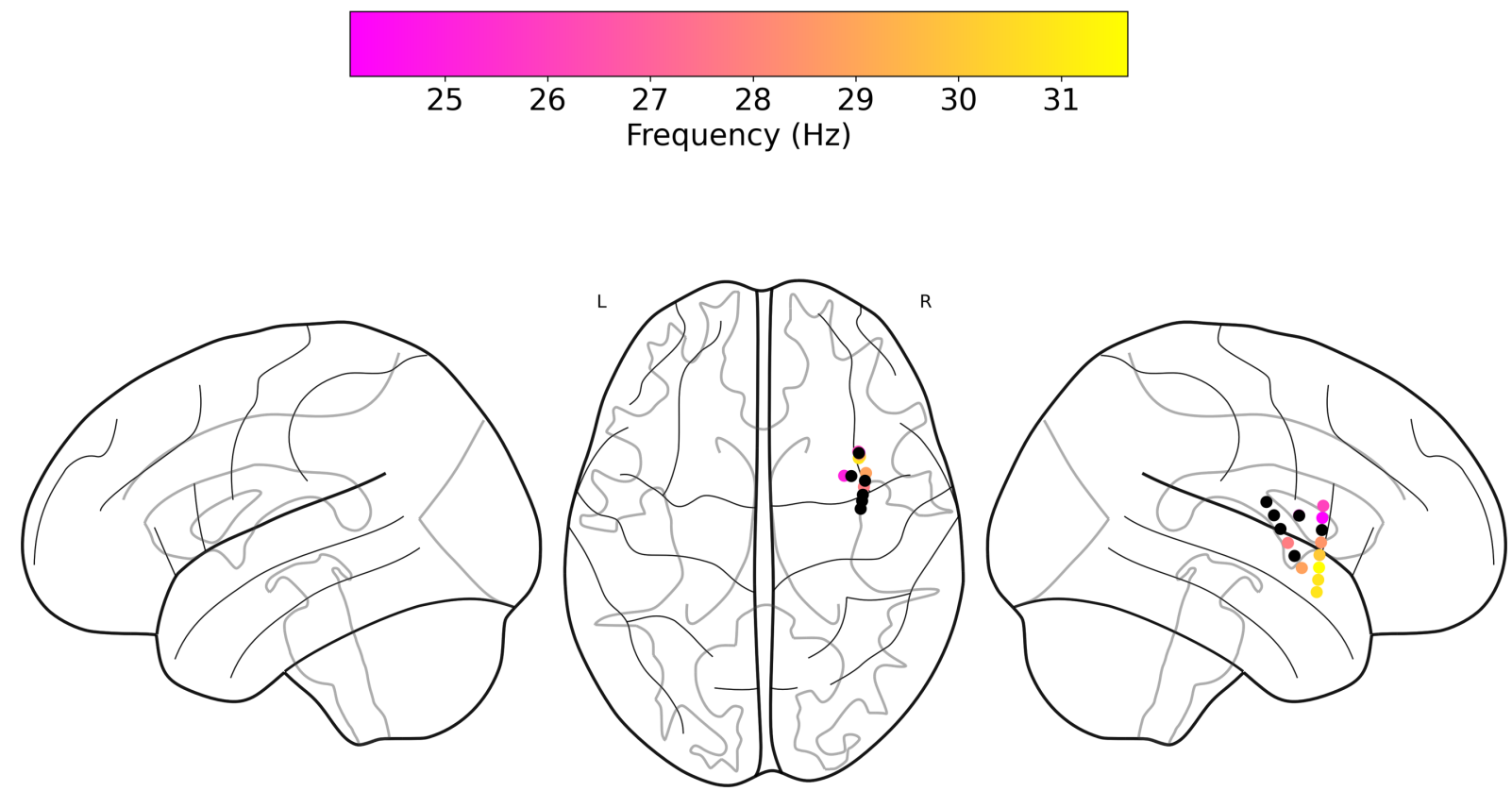

Subject \# 5, cluster \# 1, cluster frequency $=28.368 \mathrm{~Hz}, \mathrm{n}=10$
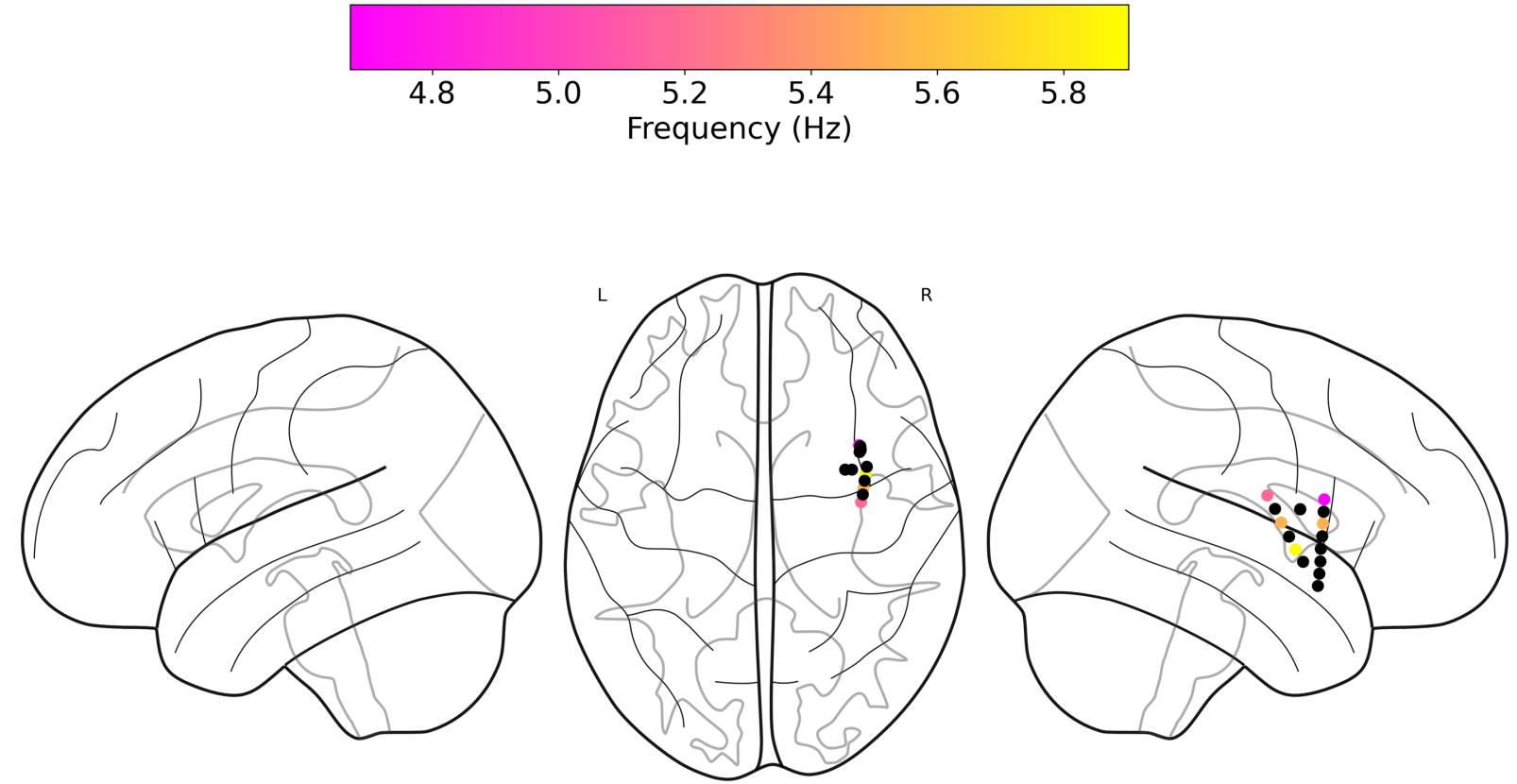

Subject \# 5, cluster \# 2, cluster frequency $=5.363 \mathrm{~Hz}, \mathrm{n}=5$ 
bioRxiv preprint doi: $\mathrm{https}$ //doi.org/10.1101/2021.12 16.473034; this version posted December 19, 2021. The copyright holder for this preprint (which was not certified by peer review) is the author/funder, who has granted bioRxiv a license to display the preprint in perpetuity. It is made available under aCC-BY-NC-ND 4.0 International license.

\section{Figure S5: Oscillation clusters in participant \#6.}
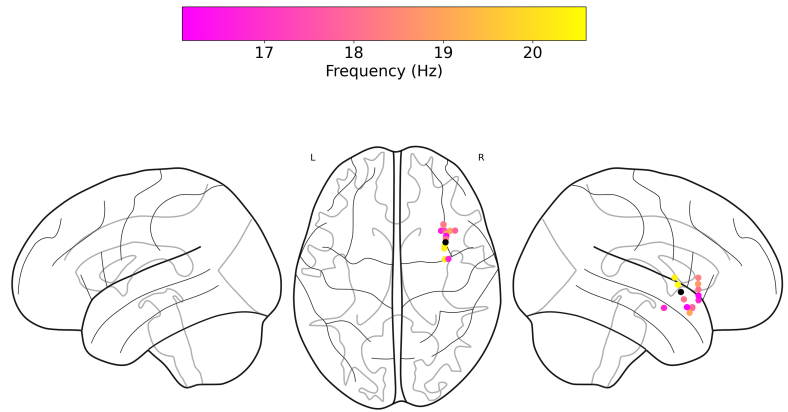

Subject \# 6, cluster \# 1, cluster frequency $=18.123 \mathrm{~Hz}, \mathrm{n}=16$
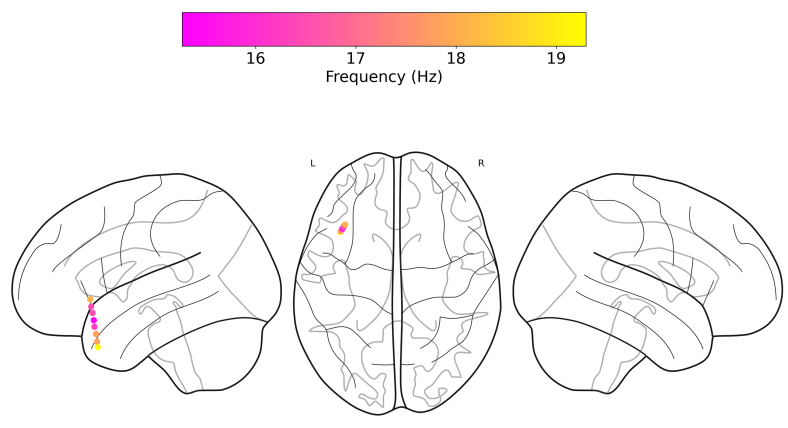

Subject \# 6, cluster \# 3, cluster frequency $=17.205 \mathrm{~Hz}, \mathrm{n}=8$
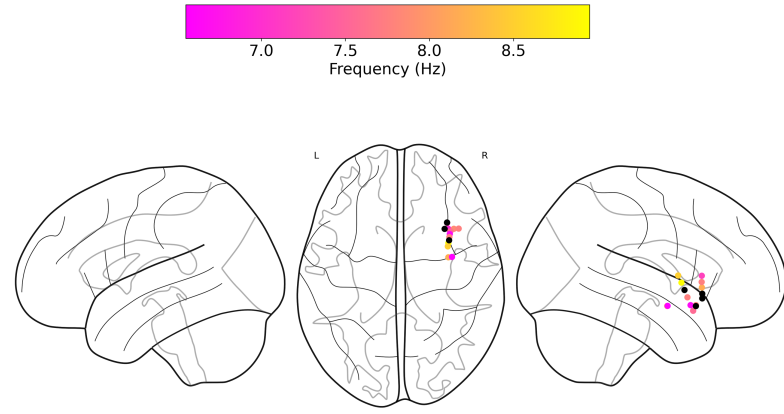

Subject \# 6, cluster \# 2, cluster frequency $=7.673 \mathrm{~Hz}, \mathrm{n}=13$

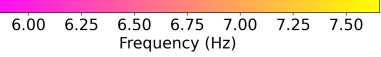

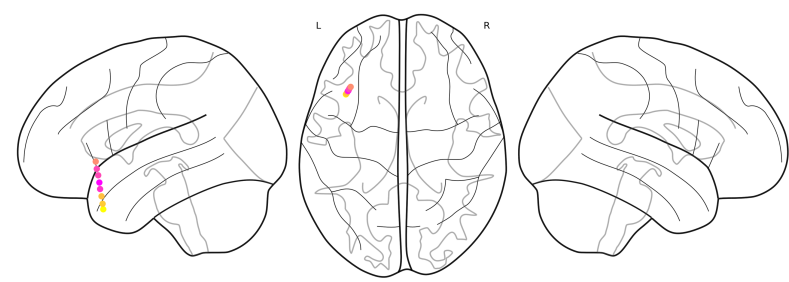

Subject \# 6, cluster \# 4, cluster frequency $=6.675 \mathrm{~Hz}, \mathrm{n}=8$ 
bioRxiv preprint doi: https://doi.org/10.1101/2021.12.16.473034; this version posted December 19, 2021. The copyright holder for this preprint (which was not certified by peer review) is the author/funder, who has granted bioRxiv a license to display the preprint in perpetuity. It is made available under aCC-BY-NC-ND 4.0 International license.

Figure S6: Oscillation clusters in participant \#7.
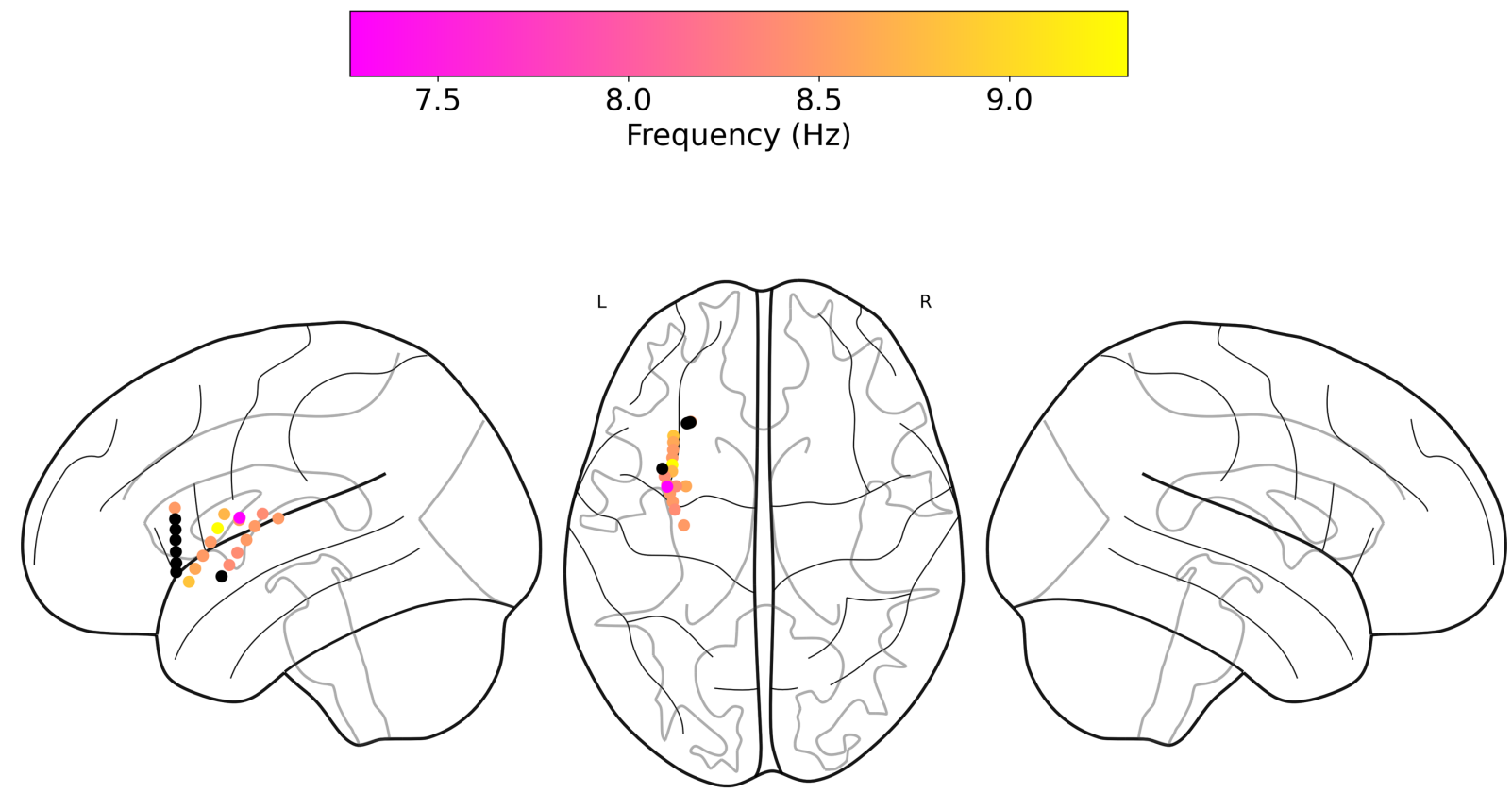

Subject \# 7, cluster \# 1, cluster frequency $=8.494 \mathrm{~Hz}, \mathrm{n}=16$
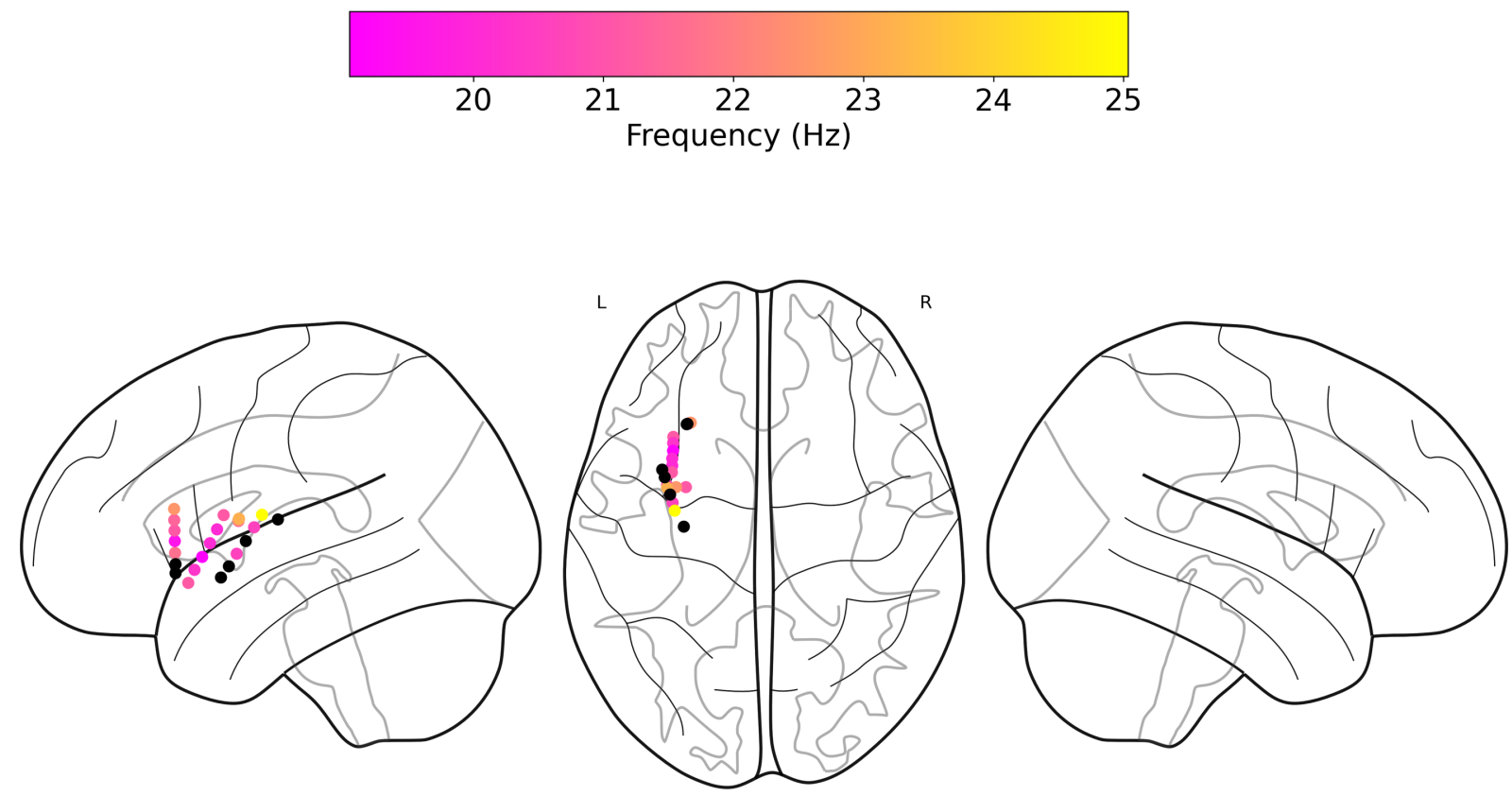

Subject \# 7, cluster \# 2, cluster frequency $=21.248 \mathrm{~Hz}, \mathrm{n}=17$ 
bioRxiv preprint doi: $\mathrm{https}$ //doi.org/10.1101/2021.12 16.473034; this version posted December 19, 2021. The copyright holder for this preprint (which was not certified by peer review) is the author/funder, who has granted bioRxiv a license to display the preprint in perpetuity. It is made available under aCC-BY-NC-ND 4.0 International license.

\section{Figure S7: Oscillation clusters in participant \#8.}

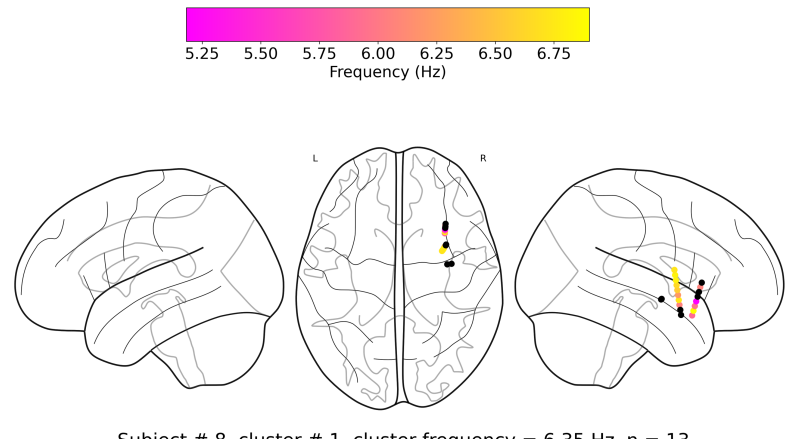

Subject \# 8, cluster \# 1, cluster frequency $=6.35 \mathrm{~Hz}, \mathrm{n}=13$

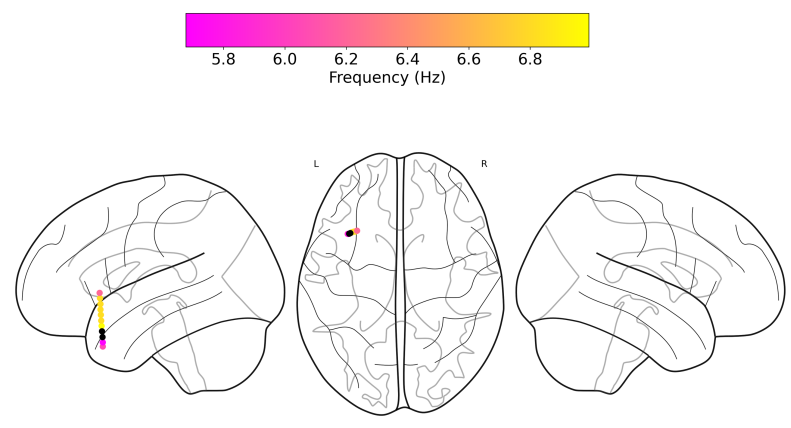

Subject \# 8, cluster \# 3, cluster frequency $=6.556 \mathrm{~Hz}, \mathrm{n}=9$

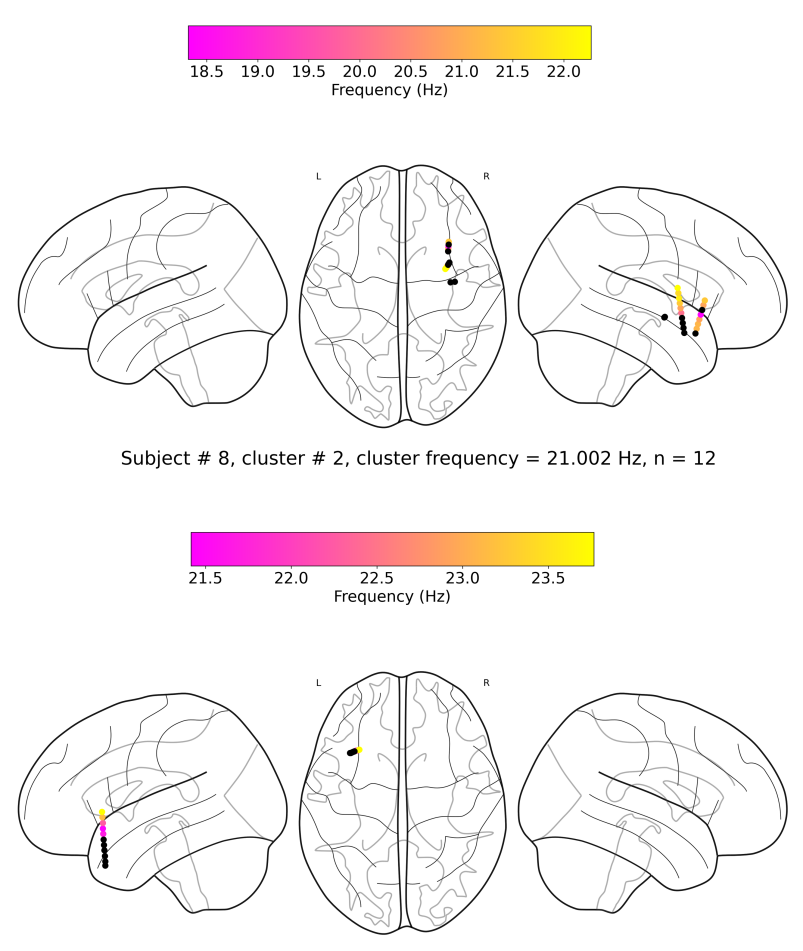

Subject \# 8, cluster \# 4, cluster frequency $=22.459 \mathrm{~Hz}, \mathrm{n}=5$ 
bioRxiv preprint doi: https://doi.org/10.1101/2021.12.16.473034; this version posted December 19, 2021. The copyright holder for this preprint (which was not certified by peer review) is the author/funder, who has granted bioRxiv a license to display the preprint in perpetuity. It is made available under aCC-BY-NC-ND 4.0 International license.

\section{Figure S8: Oscillation clusters in participant \#9.}

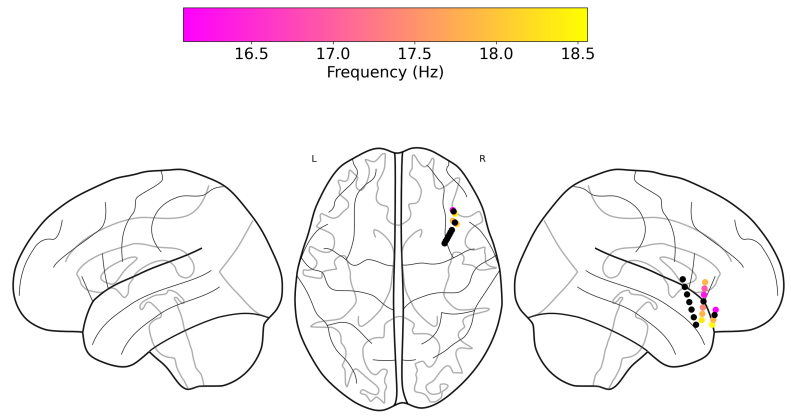

Subject \# 9, cluster \# 1, cluster frequency $=17.458 \mathrm{~Hz}, \mathrm{n}=9$

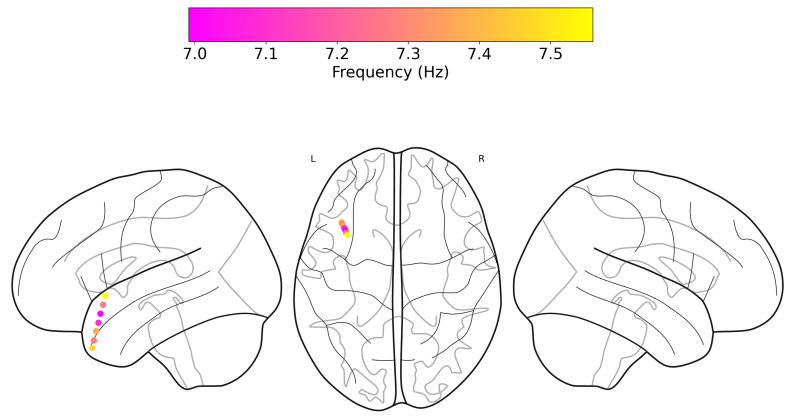

Subject \# 9, cluster \# 2, cluster frequency $=7.285 \mathrm{~Hz}, \mathrm{n}=7$

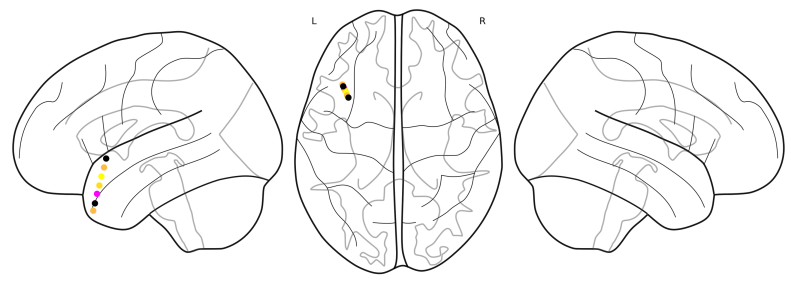

Subject \# 9, cluster \# 3, cluster frequency $=16.722 \mathrm{~Hz}, \mathrm{n}=5$ 
bioRxiv preprint doi: $\mathrm{https}$ //doi.org/10.1101/2021.12 16.473034; this version posted December 19,2021 . The copyright holder for this preprint (which was not certified by peer review) is the author/funder, who has granted bioRxiv a license to display the preprint in perpetuity. It is made available under aCC-BY-NC-ND 4.0 International license.

\section{Figure S9: Oscillation clusters in participant \#10.}

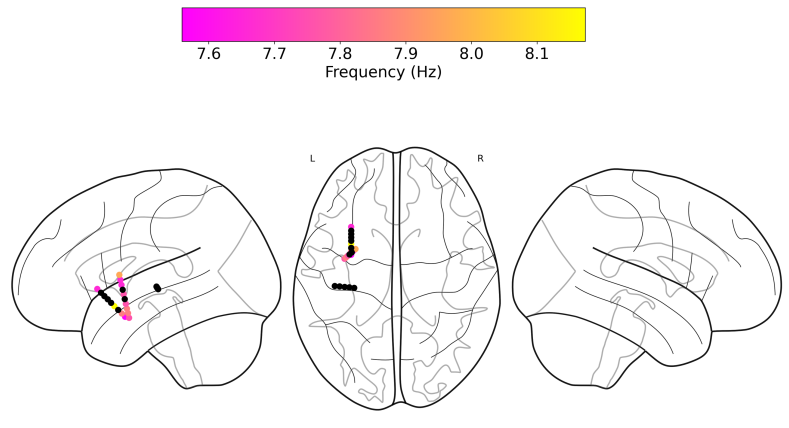

Subject \# 10, cluster \# 1, cluster frequency $=7.793 \mathrm{~Hz}, \mathrm{n}=12$
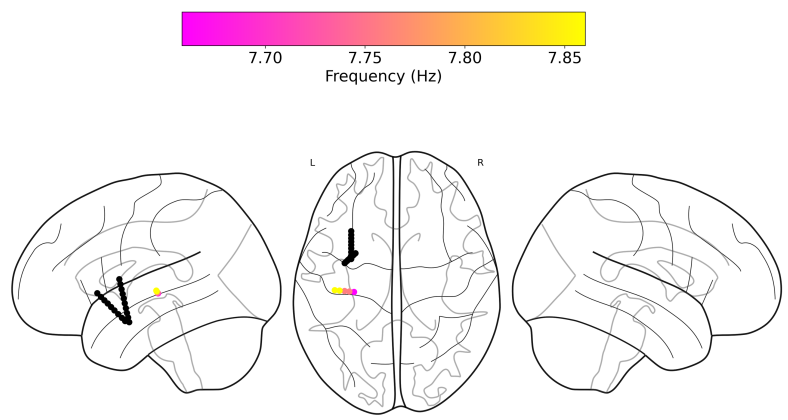

Subject \# 10, cluster \# 3, cluster frequency $=7.779 \mathrm{~Hz}, \mathrm{n}=5$

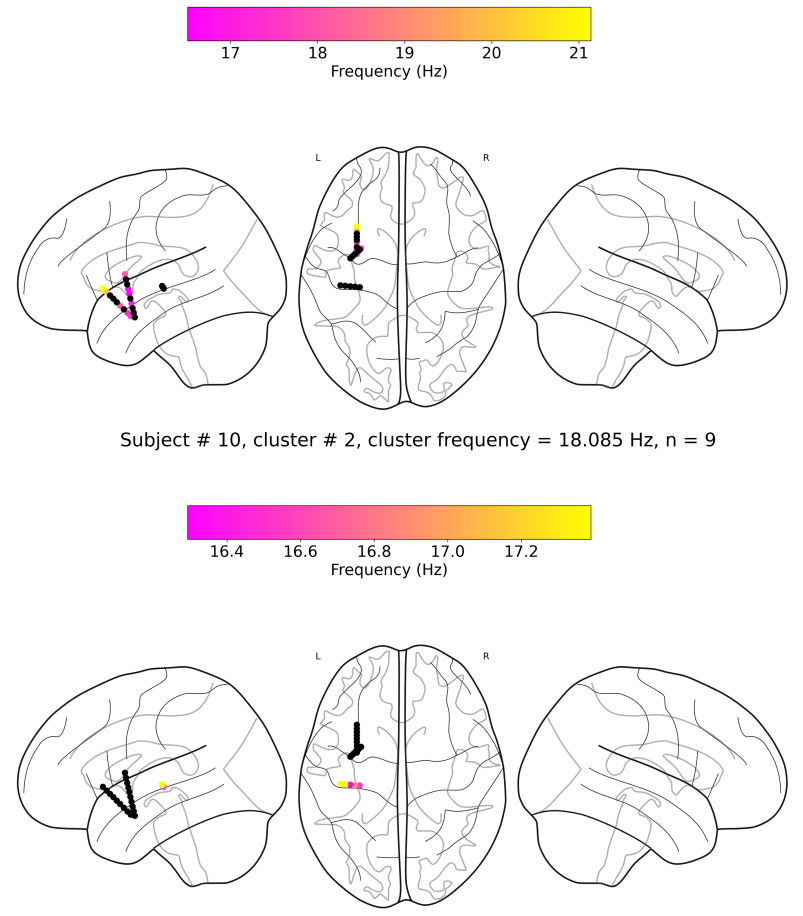

Subject \# 10, cluster \# 4, cluster frequency $=16.903 \mathrm{~Hz}, \mathrm{n}=5$ 


\section{Supplementary tables}

Table S1. Wave-strength (denoted as $\rho_{a d j}^{2}$ ) of traveling waves is positively correlated with Hilberttransformed amplitude of signals. We estimated the correlation between the average amplitude of signals of the electrodes and the wave-strength of traveling waves for each oscillation cluster. We used a circular-shuffling procedure to test the significance of correlations where we circularly shuffle the wavestrength values with respect to the average amplitude and estimate the correlation on this shuffled data to build a distribution of surrogate correlation values against which the observed correlation was tested $(p<$ 0.05 ) (Methods). We found that for $\sim 71 \%$ of clusters (20 out of the 28 clusters), there was a statistically significant correlation between amplitude and wave-strength. All, except one, clusters out of these 20 clusters, showed a statistically significant positive amplitude- $\rho_{a d j}^{2}$ correlation. Cluster frequency for an oscillation cluster was defined to be the average of the oscillation frequencies across all electrodes for the given oscillation cluster.

\begin{tabular}{|c|c|c|c|c|}
\hline Subject \# & Cluster \# & $\begin{array}{l}\text { Cluster } \\
\text { frequency }\end{array}$ & $\begin{array}{l}\text { Amplitude- } \rho_{a d j}^{2} \\
\text { Pearson } r\end{array}$ & $\begin{array}{l}<<0.05 ? \\
(\operatorname{Yes}(\mathrm{Y}) / \mathrm{No}(\mathrm{N}))\end{array}$ \\
\hline 1 & 1 & 8.08 & 0.182 & $Y$ \\
\hline 1 & 2 & 12.97 & 0.028 & $\mathrm{~N}$ \\
\hline 1 & 3 & 7.61 & -0.019 & $\mathrm{~N}$ \\
\hline 1 & 4 & 15.88 & 0.064 & $\mathrm{Y}$ \\
\hline 2 & 1 & 8.14 & 0.130 & $Y$ \\
\hline 2 & 2 & 16.69 & 0.154 & $Y$ \\
\hline 2 & 3 & 4.66 & 0.020 & $\mathrm{~N}$ \\
\hline 3 & 1 & 21.98 & 0.208 & $\mathrm{Y}$ \\
\hline 3 & 2 & 6.94 & 0.119 & $Y$ \\
\hline 5 & 1 & 28.37 & 0.097 & $\mathrm{Y}$ \\
\hline 5 & 2 & 5.36 & 0.022 & $\mathrm{~N}$ \\
\hline 6 & 1 & 18.12 & 0.012 & $\mathrm{~N}$ \\
\hline 6 & 2 & 7.67 & 0.068 & $Y$ \\
\hline 6 & 3 & 17.21 & 0.086 & $Y$ \\
\hline 6 & 4 & 6.68 & 0.181 & $Y$ \\
\hline 7 & 1 & 8.49 & 0.013 & $\mathrm{~N}$ \\
\hline 7 & 2 & 21.25 & 0.192 & $Y$ \\
\hline 8 & 1 & 6.35 & 0.118 & $\mathrm{Y}$ \\
\hline 8 & 2 & 21.00 & 0.260 & $\mathrm{Y}$ \\
\hline 8 & 3 & 6.56 & 0.057 & $\mathrm{Y}$ \\
\hline 8 & 4 & 22.46 & 0.169 & $Y$ \\
\hline 9 & 1 & 17.46 & 0.203 & $\mathrm{Y}$ \\
\hline 9 & 2 & 7.29 & 0.173 & $Y$ \\
\hline 9 & 3 & 16.72 & 0.076 & $\mathrm{Y}$ \\
\hline 10 & 1 & 7.79 & -0.080 & $Y$ \\
\hline 10 & 2 & 18.08 & 0.071 & $Y$ \\
\hline 10 & 3 & 7.78 & 0.006 & $\mathrm{~N}$ \\
\hline 10 & 4 & 16.90 & -0.028 & $\mathrm{~N}$ \\
\hline
\end{tabular}


Table S2. Wave-strength (denoted as $\rho_{a d j}^{2}$ ) of high (beta) frequency traveling waves is uncorrelated with the wave-strength $\left(\rho_{a d j}^{2}(\mathrm{low})-\rho_{a d j}^{2}(\right.$ high $\left.)\right)$, phase (phase (low)- $\rho_{a d j}^{2}$ (high)), and power (power (low)- $\rho_{a d j}^{2}$ (high)) of low (theta) frequency traveling waves. For testing the statistical significance of the observed correlations, values from one time-series were circularly shuffled with respect to the other so that the instantaneous correlation between the two time-series is destroyed, and correlation analysis was repeated on this shuffled data to build a distribution of surrogate correlation values against which the observed correlation was tested ( $p$ $<0.05)$ (Methods).

\begin{tabular}{|c|c|c|c|c|c|c|c|c|}
\hline Subject \# & $\begin{array}{l}\text { Cluster- } \\
\text { pair \# }\end{array}$ & $\begin{array}{l}\text { Cluster-pair } \\
\text { frequencies } \\
\text { (low (theta), } \\
\text { high (beta)) }\end{array}$ & $\begin{array}{l}\rho_{a d j}^{2}(\text { low })- \\
\rho_{a d j}^{2}(\text { high }) \\
\text { Pearson } r\end{array}$ & $\begin{array}{l}p<0.05 ? \\
(\mathrm{Y} / \mathrm{N})\end{array}$ & $\begin{array}{l}\text { Phase (low)- } \\
\rho_{a d j}^{2} \text { (high) } \\
\text { Pearson } r\end{array}$ & $\begin{array}{l}p<0.05 ? \\
(\mathrm{Y} / \mathrm{N})\end{array}$ & $\begin{array}{l}\text { Power (low)- } \\
\rho_{a d j}^{2}(\text { high) } \\
\text { Pearson } r\end{array}$ & $\begin{array}{l}p<0.05 ? \\
(\mathrm{Y} / \mathrm{N})\end{array}$ \\
\hline 1 & 1,4 & $8.08,15.88$ & 0.009 & $\mathrm{~N}$ & 0.012 & $\mathrm{~N}$ & 0.00001 & $\mathrm{~N}$ \\
\hline 1 & 3,2 & $7.61,12.97$ & -0.025 & $\mathrm{~N}$ & 0.034 & $\mathrm{~N}$ & -0.00017 & $\mathrm{~N}$ \\
\hline 2 & 1,2 & $8.14,16.69$ & 0.047 & $\mathrm{Y}$ & 0.024 & $\mathrm{~N}$ & -0.00014 & $\mathrm{~N}$ \\
\hline 3 & 2,1 & $6.94,21.98$ & 0.023 & $\mathrm{~N}$ & 0.045 & $\mathrm{~N}$ & -0.00014 & $\mathrm{~N}$ \\
\hline 5 & 2,1 & $5.36,28.37$ & -0.016 & $\mathrm{~N}$ & 0.054 & $\mathrm{~N}$ & -0.00018 & $\mathrm{~N}$ \\
\hline 6 & 2,1 & $7.67,18.12$ & -0.011 & $\mathrm{~N}$ & 0.061 & $\mathrm{~N}$ & -0.00031 & $\mathrm{~N}$ \\
\hline 6 & 4,3 & $6.68,17.21$ & 0.002 & $\mathrm{~N}$ & 0.051 & $\mathrm{~N}$ & -0.00016 & $\mathrm{~N}$ \\
\hline 7 & 1,2 & $8.49,21.25$ & -0.016 & $\mathrm{~N}$ & 0.040 & $\mathrm{~N}$ & 0.00010 & $\mathrm{~N}$ \\
\hline 8 & 1,2 & $6.35,21.00$ & 0.017 & $\mathrm{~N}$ & 0.028 & $\mathrm{~N}$ & -0.00001 & $\mathrm{~N}$ \\
\hline 8 & 3,4 & $6.56,22.46$ & -0.009 & $\mathrm{~N}$ & 0.035 & $\mathrm{~N}$ & 0.00012 & $\mathrm{~N}$ \\
\hline 9 & 2,3 & $7.29,16.72$ & 0.036 & $\mathrm{Y}$ & 0.030 & $\mathrm{~N}$ & -0.00015 & $\mathrm{~N}$ \\
\hline 10 & 1,2 & $7.79,18.08$ & 0.001 & $\mathrm{~N}$ & 0.011 & $\mathrm{~N}$ & -0.00002 & $\mathrm{~N}$ \\
\hline 10 & 3,4 & $7.78,16.90$ & 0.007 & $\mathrm{~N}$ & 0.016 & $\mathrm{~N}$ & 0.00010 & $\mathrm{~N}$ \\
\hline
\end{tabular}

